\title{
A new class of multi-substituted oxazole derivatives: Synthesis and antimicrobial activity
}

\author{
A BABUL REDDY ${ }^{\mathrm{a}, \mathrm{c}}$, R V HYMAVATHI ${ }^{\mathrm{b}}$ and G NARAYANA SWAMY ${ }^{\mathrm{a}, *}$ \\ ${ }^{a}$ Department of Chemistry, ${ }^{b}$ Deptartment of Bio-Chemistry, Sri Krishnadevaraya University, \\ Anantapur 515 055, India \\ ${ }^{\mathrm{c}}$ Department of Molecular Science and Technology, Ajou University, San 5, Woncheon-dong, \\ Yeongtong, Suwon 443-749, South Korea \\ e-mail: narayanaswamy.golla@gmail.com
}

MS received 14 October 2012; revised 30 December 2012; accepted 21 January 2013

\begin{abstract}
A new and efficient method for the synthesis of multi-substituted oxazole derivatives from various aldehydes has been described. Twenty novel multi-substituted oxazoles containing a heterocyclic moiety were synthesized and evaluated for their antimicrobial activity. The prepared compounds are all reported for the first time and their structures were established by elemental analysis, IR, ${ }^{1} \mathrm{H}-\mathrm{NMR}$, and ${ }^{13} \mathrm{C}-\mathrm{NMR}$ and Mass spectra. All the synthesized compounds exhibited in vitro antimicrobial activity.
\end{abstract}

Keywords. Oxazoles; antimicrobial activity.

\section{Introduction}

Oxazoles are a common structural motif found in numerous molecules that display antiviral, antifungal, antibacterial, and antiproliferative activities. ${ }^{1,2}$ In particular, many efforts have been focused for synthesis of substituted oxazoles, due to their existence in substructures of many pharmaceuticals. ${ }^{3-5}$ The most common methods for the preparation of substituted oxazoles include Negishi cross-coupling, ${ }^{6}$ Ritter reactions of $\alpha$-oxo tosylates, ${ }^{7}$ one-pot Van Leusen synthesis of 4,5-disubstituted oxazoles in ionic liquids, ${ }^{8}$ Nickel-catalysed synthesis of oxazoles, ${ }^{9}$ transformation of 4-bis(methylthio)methylene-2-phenyloxazol-5one, ${ }^{10}$ one-pot Friedel-Crafts/Robinson-Gabriel synthesis of oxazoles, ${ }^{11}$ reaction of $\beta$-(acyloxy)vinyl azides with $(\mathrm{EtO})_{3} \mathrm{P}^{12}$ reaction of benzoin carboxylates with formamide, ${ }^{13}$ and decarboxylation of $N$ acylisoxazol-5-ones by means of photolysis or pyrolysis. ${ }^{14,15}$ Direct conversion of ketones to the corresponding oxazoles has received little attention and there are only few studies published. For example, 2-alkyl-5aryloxazoles were prepared by the direct reactions of aromatic methyl ketones and nitriles with $\mathrm{Tl}(\mathrm{OAc})_{3}{ }^{16}$ or iodobenzene diacetate ${ }^{17}$ in the presence of trifluoromethanesulphonic acid (TfOH). Furthermore, very recently, an expedient method for the direct conversion of aldehydes to substituted oxazoles was presented, ${ }^{18,19}$

*For correspondence but products were only disubstituted oxazoles. However, these methods have been applied only for few aromatic aldehydes, which limit their practical applications. We therefore, developed a new and efficient method for the synthesis of multi-substituted oxazoles at room temperature.

\section{Experimental}

All melting points were determined in open capillary tubes on Mel-Temp apparatus (Laboratory Devices, Cambridge, MA), and are uncorrected. IR spectra were recorded as $\mathrm{KBr}$ pellets on a Perkin-Elmer 283 double beam spectrophotometer in $\mathrm{cm}^{-1} \cdot{ }^{1} \mathrm{H}$ and ${ }^{13} \mathrm{C}$ NMR spectra were recorded on Mercury-400 spectrophotometer operating at $400 \mathrm{MHz}$ for ${ }^{1} \mathrm{H}$, and $100 \mathrm{MHz}$ for ${ }^{13} \mathrm{C}$ using DMSO- $\mathrm{d}_{6}$ and $\mathrm{CDCl}_{3}$ as solvent; chemical shifts were referenced to TMS. All compounds were characterized by the above techniques.

\subsection{Preparation of compound 2-benzo[d][1,3]dioxol-} 5-yl)-2-(tert-butyldimethylsilyloxy)acetonitrile (2)

A solution of benzo[ $d][1,3]$ dioxole-5-carbaldehyde (1; $25 \mathrm{~g}, 166 \mathrm{mmol}$ ) in $\mathrm{MeCN}$ was added drop-wise to a stirring mixture of $\mathrm{KCN}(43.3 \mathrm{~g}, 666 \mathrm{mmol}), \mathrm{TBSCl}$ $(25 \mathrm{~g}, 166 \mathrm{mmol})$ and $\mathrm{ZnI}_{2}(0.8 \mathrm{~g}, 1.7 \mathrm{mmol})$ at room temperature and the mixture was stirred at this temperature for overnight. The mixture was diluted with $\mathrm{H}_{2} \mathrm{O}$ 
and extracted with EtOAc $(3 \times 50 \mathrm{~mL})$. The organic extract was washed with brine and dried over anhydrous $\mathrm{Na}_{2} \mathrm{SO}_{4}$. The solvent was removed in vacuo and the residue was purified by silica gel (100-200 mesh) column chromatography (CC) using EtOAc-petroleum ether (5:1) as eluent affording $\mathbf{2}$ as pale brown liquid in $85 \%$ yield. IR $\left(\mathrm{KBr}, \mathrm{cm}^{-1}\right): 3184,2812,2157,1975$, 1254. ${ }^{1} \mathrm{H}$ NMR $\left(\mathrm{CDCl}_{3}, 400 \mathrm{MHz}\right) \delta: 0.24(6 \mathrm{H}, \mathrm{s}), 0.98$ $(9 \mathrm{H}, \mathrm{s}), 5.42(1 \mathrm{H}, \mathrm{s}), 6.02(2 \mathrm{H}, \mathrm{s}), 6.86(1 \mathrm{H}, \mathrm{d}, J=$ $6.4 \mathrm{~Hz}), 7.03(1 \mathrm{H}, \mathrm{d}, J=5.6 \mathrm{~Hz}), 7.27(1 \mathrm{H}, \mathrm{s}) .{ }^{13} \mathrm{C}$ NMR $\left(\mathrm{CDCl}_{3}, 100 \mathrm{MHz}\right): \delta(\mathrm{ppm}) 25.94\left(\mathrm{CH}_{3}\right), 30.54$ (C), $69.25(\mathrm{CH}), 102.32\left(\mathrm{CH}_{2}\right), 112.74(\mathrm{CH}), 114.58$ $(\mathrm{CH}), 119.53(\mathrm{CN}), 1223.17(\mathrm{CH}), 128.69(\mathrm{C}), 148.75$ (C), 148.93 (C). MS (ESI, $m / z): 292.5\left[\mathrm{M}^{+}+1\right]$. Anal. Calcd for $\mathrm{C}_{15} \mathrm{H}_{21} \mathrm{NO}_{3} \mathrm{Si}$ (291.42): C, 61.84; H, 7.27; N, 4.85. Found: C, 61.82; H, 7.26; N, 4.83.

\subsection{General procedure for synthesis of compounds $(3 a-t)$}

$n$-BuLi (1.6 M in hexane, 1.1 eq.) was added dropwise to a solution of DPA (1.1 eq.) in THF at $-78^{\circ} \mathrm{C}$ under an inert atmosphere, the pale yellow solution was warmed to $0^{\circ} \mathrm{C}$ and stirred at this temperature for $30 \mathrm{~min}$. The LDA solution thus prepared was again cooled to $-78^{\circ} \mathrm{C}$ and a solution of compound 2 in THF was added drop-wise over a period of $1.5 \mathrm{~h}$ under an inert atmosphere. The resulting mixture was stirred at $-78^{\circ} \mathrm{C}$ temperature for $30 \mathrm{~min}$, and then solution of the respective aldehydes ( 0.8 eq.) in THF was added at $-78^{\circ} \mathrm{C}$. The mixture was stirred at same temperature for $8-12 \mathrm{~h}$ under an inert atmosphere. The reaction was quenched by addition of ice-cold $\mathrm{H}_{2} \mathrm{O}$ and extracted with EtOAc. The organic extract was washed with brine and dried over anhydrous $\mathrm{Na}_{2} \mathrm{SO}_{4}$, prior to concentration under reduced pressure. The residue was purified by CC over silica gel (60-120 mesh) adsorbent, using EtOAc -petroleum ether to get compound 3.

2.2a 1-Benzo[d][1,3]dioxol-5-yl)-2-(tertbutyldimethylsilyloxy)-2-(furan-3-yl)ethanone (3a): Yield: $85 \%$, $\mathrm{mp}: 155-157^{\circ} \mathrm{C}$. IR $\left(\mathrm{KBr}, \mathrm{cm}^{-1}\right)$ : 3433, 3141, 2262, 1724, 1254. ${ }^{1} \mathrm{H}$ NMR $\left(\mathrm{CDCl}_{3}\right) \delta: 0.26(6 \mathrm{H}, \mathrm{s}), 0.96$ $(9 \mathrm{H}, \mathrm{s}), 4.92(1 \mathrm{H}, \mathrm{s}), 6.03(1 \mathrm{H}, \mathrm{s}), 6.11(2 \mathrm{H}, \mathrm{s}), 6.58$ $(1 \mathrm{H}, \mathrm{d}, J=19.6 \mathrm{~Hz}), 7.16(1 \mathrm{H}, \mathrm{d}, J=3.6 \mathrm{~Hz}), 7.27$ $(1 \mathrm{H}, \mathrm{d}, J=12 \mathrm{~Hz}), 7.46(1 \mathrm{H}, \mathrm{d}, J=6.4 \mathrm{~Hz}), 7.59(1 \mathrm{H}$, s), $7.78(1 \mathrm{H}, \mathrm{s}) .{ }^{13} \mathrm{C} \mathrm{NMR}\left(\mathrm{CDCl}_{3}\right) \delta:-2.83\left(\mathrm{CH}_{3}\right)$, $26.02\left(\mathrm{CH}_{3}\right), 31.55(\mathrm{C}), 87.66(\mathrm{CH}), 101.02\left(\mathrm{CH}_{2}\right)$, $108.74(\mathrm{CH}), 110.41(\mathrm{CH}), 112.74(\mathrm{CH}), 121.14(\mathrm{C})$, $123.79(\mathrm{CH}), 143.75(\mathrm{CH}), 148.43(\mathrm{C}), 153.37(\mathrm{C})$, $194.63(\mathrm{C}=\mathrm{O})$. MS (ESI, $m / z): 361.2\left[\mathrm{M}^{+}+1\right]$. Anal. calcd for $\mathrm{C}_{19} \mathrm{H}_{24} \mathrm{O}_{5} \mathrm{Si}$ (360.44): C 63.29, H 6.67; found C 63.32, H 6.74.

2.2b 1-Benzo[d][1,3]dioxol-5-yl)-2-(tertbutyldimethylsilyloxy)-2-(furan-2-yl)ethanone (3b): Yield: 82\%, mp: $147-148^{\circ} \mathrm{C}$. IR $\left(\mathrm{KBr}, \mathrm{cm}^{-1}\right): 3421,3044,2243$, 1712, 1215. ${ }^{1} \mathrm{H} \mathrm{NMR}\left(\mathrm{CDCl}_{3}\right) \delta: 0.26(6 \mathrm{H}, \mathrm{s}), 0.98$ $(9 \mathrm{H}, \mathrm{s}), 4.86(1 \mathrm{H}, \mathrm{s}), 6.03(2 \mathrm{H}, \mathrm{s}), 6.83(1 \mathrm{H}, \mathrm{d}, J=$ $5.6 \mathrm{~Hz}), 7.05(1 \mathrm{H}, \mathrm{d}, J=2.8 \mathrm{~Hz}), 7.35(1 \mathrm{H}, \mathrm{t}, J=$ $16.4 \mathrm{~Hz}), 7.56(1 \mathrm{H}, \mathrm{d}, J=12.6 \mathrm{~Hz}) .7 .78(1 \mathrm{H}, \mathrm{d}, J=$ $10.8 \mathrm{~Hz}) .{ }^{13} \mathrm{C}$ NMR $\left(\mathrm{CDCl}_{3}\right) \delta:-2.63\left(\mathrm{CH}_{3}\right), 25.91$ $\left(\mathrm{CH}_{3}\right), 39.55(\mathrm{C}), 89.68(\mathrm{CH}), 102.32\left(\mathrm{CH}_{2}\right), 108.74$ $(\mathrm{CH}), 111.41(\mathrm{CH}), 125.17(\mathrm{CH}), 126.69(\mathrm{C}), 148.75$ (C), $153.97(\mathrm{C}), 196.66(\mathrm{C}=\mathrm{O})$. MS (ESI, $m / z): 361.04$ $\left[\mathrm{M}^{+}+1\right]$. Anal. Calcd for: $\mathrm{C}_{21} \mathrm{H}_{32} \mathrm{O}_{3} \mathrm{Si}$ (360.54): $\mathrm{C}$ 66.89, H 8.57; found C 66.82, H 8.62.

2.2c 1-Benzo[d][1,3]dioxol-5-yl)-2-(tertbutyldimethylsilyloxy)-2-(thiophen-2-yl)ethanone (3c): Yield: 88\%, Semi solid. IR $\left(\mathrm{KBr}, \mathrm{cm}^{-1}\right)$ : 3341, 3120, 2255, 1755, 1288. ${ }^{1} \mathrm{H}$ NMR $\left(\mathrm{CDCl}_{3}\right) \delta: 0.24(6 \mathrm{H}, \mathrm{s}), 0.99(9 \mathrm{H}, \mathrm{s})$, $4.75(1 \mathrm{H}, \mathrm{s}), 6.03(2 \mathrm{H}, \mathrm{s}), 6.82(1 \mathrm{H}, \mathrm{d}, J=5.6 \mathrm{~Hz})$, $7.18(1 \mathrm{H}, \mathrm{d}, J=3.2 \mathrm{~Hz}), 7.43(1 \mathrm{H}, \mathrm{t}, J=3.2,5.6 \mathrm{~Hz})$, $7.52(1 \mathrm{H}, \mathrm{s}), 7.74(1 \mathrm{H}, \mathrm{d}, J=8.0 \mathrm{~Hz}) 7.86(1 \mathrm{H}, \mathrm{d}, J$ $=12.4 \mathrm{~Hz}) .{ }^{13} \mathrm{C} \mathrm{NMR}\left(\mathrm{CDCl}_{3}\right) \delta:-2.81\left(\mathrm{CH}_{3}\right), 25.97$ $\left(\mathrm{CH}_{3}\right), 26.01\left(\mathrm{CH}_{2}\right), 39.54(\mathrm{C}), 89.67(\mathrm{CH}), 102.34$ $\left(\mathrm{CH}_{2}\right), 108.73(\mathrm{CH}), 111.42(\mathrm{CH}), 125.17(\mathrm{CH}), 126.64$ (C), $148.71(\mathrm{C}), 153.93(\mathrm{C}), 196.55(\mathrm{C}=\mathrm{O})$. MS (ESI, $m / z)$ : $377.1\left[\mathrm{M}^{+}+1\right]$. Anal. calcd for $\mathrm{C}_{19} \mathrm{H}_{24} \mathrm{O}_{4} \mathrm{SSi}$ (376.54): C 60.69, H 6.47, S 8.52; found C 60.82, H $6.45, \mathrm{~S} 8.55$.

2.2d 1-Benzo[d][1,3]dioxol-5-yl)-2-(tertbutyldimethylsilyloxy)-2-(pyridin-4-yl)-ethanone (3d): Yield: 87\%, mp: $171-172^{\circ} \mathrm{C}$. IR $\left(\mathrm{KBr}, \mathrm{cm}^{-1}\right): 3427,3014,2214$, 1711, 1657. ${ }^{1} \mathrm{H} \mathrm{NMR}\left(\mathrm{CDCl}_{3}\right) \delta: 0.27(6 \mathrm{H}, \mathrm{s}), 0.97(9 \mathrm{H}$, s), $4.95(1 \mathrm{H}, \mathrm{s}), 6.07(2 \mathrm{H}, \mathrm{s}), 7.13(1 \mathrm{H}, \mathrm{d}, J=8.0 \mathrm{~Hz})$, $7.46(1 \mathrm{H}, \mathrm{s}), 7.49(1 \mathrm{H}, \mathrm{d}, J=4.8 \mathrm{~Hz}), 7.65(2 \mathrm{H}, \mathrm{d}, J=$ $8.8 \mathrm{~Hz}), 8.52(2 \mathrm{H}, \mathrm{d}, J=6.4 \mathrm{~Hz}) .{ }^{13} \mathrm{C}$ NMR $\left(\mathrm{CDCl}_{3}\right)$ $\delta$ : $-2.46\left(\mathrm{CH}_{3}\right), 25.91\left(3 \mathrm{CH}_{3}\right), 30.19(\mathrm{C}), 91.54(\mathrm{CH})$, $102.38\left(\mathrm{CH}_{2}\right), 109.72(\mathrm{CH}), 111.99(\mathrm{CH}), 124.55(\mathrm{CH})$, $125.22(\mathrm{CH}), 148.75(\mathrm{C}), 149.99(\mathrm{CH}), 150.35(\mathrm{CH})$, $153.91(\mathrm{C}), 195.84(\mathrm{C}=\mathrm{O})$. MS (ESI, $m / z): 372.2$ $\left[\mathrm{M}^{+}+1\right]$. Anal. calcd for $\mathrm{C}_{20} \mathrm{H}_{25} \mathrm{NO}_{4} \mathrm{Si}$ (371.50): $\mathrm{C}$ 64.69, H 6.77, N 3.76; found C 64.62, H 6.75, N 3.79.

2.2e 1-Benzo[d][1,3]dioxol-5-yl)-2-(tertbutyldimethylsilyloxy)-2-cyclohexyl-ethanone (3e): Yield: 82\%, IR $\left(\mathrm{KBr}, \mathrm{cm}^{-1}\right): 3335,3010,2231,1700,1612,1125,943$. ${ }^{1} \mathrm{H}$ NMR $\left(\mathrm{CDCl}_{3}\right) \delta: 0.23\left(6 \mathrm{H}, \mathrm{s}, \mathrm{CH}_{3}\right), 0.98(9 \mathrm{H}, \mathrm{s})$, $1.28-1.36(4 \mathrm{H}, \mathrm{q}), 1.46(4 \mathrm{H}, \mathrm{q}), 1.98(2 \mathrm{H}, \mathrm{q}), 2.54(1 \mathrm{H}$, 
q), $4.35(1 \mathrm{H}, \mathrm{d}, J=8.4 \mathrm{~Hz}), 6.03(2 \mathrm{H}, \mathrm{s}), 6.83(1 \mathrm{H}, \mathrm{d}$, $J=6.4 \mathrm{~Hz}), 7.59(1 \mathrm{H}, \mathrm{s}), 7.78(1 \mathrm{H}, \mathrm{d}, J=6.8 \mathrm{~Hz})$. ${ }^{13} \mathrm{C}$ NMR $\left(\mathrm{CDCl}_{3}\right) \delta$ : $-2.38\left(\mathrm{CH}_{3}\right), 25.82\left(2 \mathrm{CH}_{2}\right)$, $25.91\left(\mathrm{CH}_{3}\right), 26.07\left(\mathrm{CH}_{2}\right), 27.13\left(2 \mathrm{CH}_{2}\right), 39.55(\mathrm{C})$, $89.68(\mathrm{CH}), 102.32\left(\mathrm{CH}_{2}\right), 108.74(\mathrm{CH}), 111.41(\mathrm{CH})$, $125.17(\mathrm{CH}), 126.69(\mathrm{C}), 148.75(\mathrm{C}), 153.97(\mathrm{C})$, $196.66(\mathrm{C}=\mathrm{O})$. MS (ESI, $m / z)$ : $377.2\left[\mathrm{M}^{+}+1\right]$. Anal. calcd for $\mathrm{C}_{21} \mathrm{H}_{32} \mathrm{O}_{3} \mathrm{Si}$ (376.54): C 66.89, $\mathrm{H}$ 8.57; found C 66.82, H 8.62.

2.2f 1-Benzo[d][1,3]dioxol-5-yl)-2-(tertbutyldimethylsilyloxy)-2-phenylethanone (3f): Yield: 87\%, mp: 122-124 ${ }^{\circ} \mathrm{C}$. IR $\left(\mathrm{KBr}, \mathrm{cm}^{-1}\right): 3387,3074,2235,1714$, 1624. ${ }^{1} \mathrm{H} \mathrm{NMR}\left(\mathrm{CDCl}_{3}\right) \delta: 0.22(6 \mathrm{H}, \mathrm{s}), 0.99(9 \mathrm{H}, \mathrm{s})$, $5.96(1 \mathrm{H}, \mathrm{s}), 6.07(2 \mathrm{H}, \mathrm{s}), 7.09(1 \mathrm{H}, \mathrm{s}), 7.13(1 \mathrm{H}, \mathrm{d}, J$ $=6.0 \mathrm{~Hz}), 7.49(1 \mathrm{H}, \mathrm{d}, J=8.4 \mathrm{~Hz}) \cdot{ }^{13} \mathrm{C} \mathrm{NMR}\left(\mathrm{CDCl}_{3}\right)$ $\delta:-2.86\left(\mathrm{CH}_{3}\right), 25.94\left(3 \mathrm{CH}_{3}\right), 30.25(\mathrm{C}), 90.55(\mathrm{CH})$, $103.55\left(\mathrm{CH}_{2}\right), 109.77(\mathrm{CH}), 111.06(\mathrm{CH}), 127.08$ $(\mathrm{CH}), 129.29(2 \mathrm{CH}), 129.88(2 \mathrm{CH}), 132.43(\mathrm{C}), 148.75$ (C), $151.33(\mathrm{CH}), 153.94(\mathrm{C}), 196.83(\mathrm{C}=\mathrm{O})$. MS (ESI, $m / z): 371.2\left[\mathrm{M}^{+}+1\right]$. Anal. calcd for $\mathrm{C}_{21} \mathrm{H}_{26} \mathrm{NO}_{4} \mathrm{Si}$ (370.21): C 68.09, H 7.07; found C 68.02, H 7.15.

$2.2 \mathrm{~g}$ 1-Benzo[d][1,3]dioxol-5-yl)-2-(tertbutyldimethylsilyloxy)-2-(3-nitrophenyl)ethanone (3g): Yield: 80\%, mp: $110-112^{\circ} \mathrm{C}$. IR $\left(\mathrm{KBr}, \mathrm{cm}^{-1}\right)$ : 3342, 3032, 2247, $1715,1601,1542,1367,1208 .{ }^{1} \mathrm{H}$ NMR $\left(\mathrm{CDCl}_{3}\right) \delta$ : $0.23(6 \mathrm{H}, \mathrm{s}), 0.97(9 \mathrm{H}, \mathrm{s}), 5.97(1 \mathrm{H}, \mathrm{s}), 6.07(2 \mathrm{H}, \mathrm{s})$, $7.02(1 \mathrm{H}, \mathrm{s}), 7.13(1 \mathrm{H}, \mathrm{d}, J=4.0 \mathrm{~Hz}), 7.29(1 \mathrm{H}, \mathrm{d}$, $J=8.0 \mathrm{~Hz}), 7.42(1 \mathrm{H}, \mathrm{t}, J=8.4,4.6 \mathrm{~Hz}),, 7.49(1 \mathrm{H}$, $\mathrm{d}, J=4.8 \mathrm{~Hz}), 7.64(1 \mathrm{H}, \mathrm{d}, J=8.8 \mathrm{~Hz}), 7.86(1 \mathrm{H}$, $\mathrm{d}, J=12.0 \mathrm{~Hz}) \cdot{ }^{13} \mathrm{C} \mathrm{NMR}\left(\mathrm{CDCl}_{3}\right) \delta:-2.45\left(\mathrm{CH}_{3}\right)$, $25.86\left(3 \mathrm{CH}_{3}\right), 30.22(\mathrm{C}), 90.57(\mathrm{CH}), 103.54\left(\mathrm{CH}_{2}\right)$, $109.77(\mathrm{CH}), 111.07(\mathrm{CH}), 127.05(\mathrm{CH}), 129.55$ $(2 \mathrm{CH}), 129.84(\mathrm{CH}), 132.47(\mathrm{C}), 143.44(\mathrm{CH}), 148.77$ (C), 151.35 (C), $153.86(\mathrm{C}), 196.94(\mathrm{C}=\mathrm{O})$. MS (ESI, $m / z)$ : $416.1\left[\mathrm{M}^{+}+1\right]$. Anal. calcd for $\mathrm{C}_{21} \mathrm{H}_{25} \mathrm{NO}_{6} \mathrm{Si}$ (415.16): C 60.74, H 6.07, N 3.35; found C 60.02, H 6.12, N 3.41 .

2.2h 1-Benzo[d][1,3]dioxol-5-yl)-2-(tertbutyldimethylsilyloxy)-2-(2-nitrophenyl)ethanone (3h): Yield: 75\%, IR $\left(\mathrm{KBr}, \mathrm{cm}^{-1}\right)$ : 3322, 3030, 2237, 1710, 1611, 1548, 1362, 1214. ${ }^{1} \mathrm{H}$ NMR $\left(\mathrm{CDCl}_{3}\right) \delta: 0.22(6 \mathrm{H}, \mathrm{s}), 0.98$ $(9 \mathrm{H}, \mathrm{s}), 5.96(1 \mathrm{H}, \mathrm{s}), 6.07(2 \mathrm{H}, \mathrm{s}), 7.12(1 \mathrm{H}, \mathrm{s}), 7.21$ $(1 \mathrm{H}, \mathrm{d}, J=8.0 \mathrm{~Hz}), 7.34(1 \mathrm{H}, \mathrm{d}, J=4.8 \mathrm{~Hz}), 7.45$ $(1 \mathrm{H}, \mathrm{t}, J=8.8,8.0 \mathrm{~Hz}), 7.56(1 \mathrm{H}, \mathrm{d}, J=6.4 \mathrm{~Hz})$, $7.69(1 \mathrm{H}, \mathrm{d}, J=8.0 \mathrm{~Hz}), 7.82(1 \mathrm{H}, \mathrm{d}, J=6.8 \mathrm{~Hz})$. ${ }^{13} \mathrm{C} \mathrm{NMR}\left(\mathrm{CDCl}_{3}\right) \delta:-2.54\left(\mathrm{CH}_{3}\right), 25.87\left(3 \mathrm{CH}_{3}\right)$, $30.23(\mathrm{C}), 90.56(\mathrm{CH}), 103.57\left(\mathrm{CH}_{2}\right), 109.72(\mathrm{CH})$, $111.17(\mathrm{CH}), 127.10(\mathrm{CH}), 129.54(2 \mathrm{CH}), 129.78$
$(\mathrm{CH}), 132.56(\mathrm{C}), 143.58(\mathrm{CH}), 148.77(\mathrm{C}), 151.15$ (C), $153.42(\mathrm{C}), 196.87(\mathrm{C}=\mathrm{O})$. MS (ESI, $m / z)$ : 416.5 $\left[\mathrm{M}^{+}+1\right]$. Anal. calcd for $\mathrm{C}_{21} \mathrm{H}_{25} \mathrm{NO}_{6} \mathrm{Si}$ (415.16): $\mathrm{C}$ 60.74, H 6.07, N 3.35; found C 60.06, H 6.11, N 3.42 .

$2.2 \mathrm{i} \quad 1-B e n z o[d][1,3]$ dioxol-5-yl)-2-(tertbutyldimethylsilyloxy)-2-(m-tolyl)ethanone (3i): Yield: $87 \%$, mp 197-199 ${ }^{\circ} \mathrm{C}$. IR $\left(\mathrm{KBr}, \mathrm{cm}^{-1}\right)$ : $3341,3124,2252,1713$, 1602, 1214, 987. ${ }^{1} \mathrm{H}$ NMR $\left(\mathrm{CDCl}_{3}\right) \delta: 0.20(6 \mathrm{H}, \mathrm{s})$, $0.99(9 \mathrm{H}, \mathrm{s}), 3.45(3 \mathrm{H}, \mathrm{s}), 5.97(1 \mathrm{H}, \mathrm{s}), 6.07(2 \mathrm{H}, \mathrm{s})$, $7.08(1 \mathrm{H}, \mathrm{s}), 7.16(1 \mathrm{H}, \mathrm{d}, J=8.8 \mathrm{~Hz}), 7.23(1 \mathrm{H}, \mathrm{d}$, $J=6.4 \mathrm{~Hz}), 7.48(1 \mathrm{H}, \mathrm{t}, J=8.0 \mathrm{~Hz}), 7.62(1 \mathrm{H}, \mathrm{d}, J$ $=4.8 \mathrm{~Hz}), 7.73(1 \mathrm{H}, \mathrm{d}, J=2.8 \mathrm{~Hz}), 7.84(1 \mathrm{H}, \mathrm{d}, J$ $=4.8 \mathrm{~Hz}) \cdot{ }^{13} \mathrm{C}$ NMR $\left(\mathrm{CDCl}_{3}\right) \delta:-2.47\left(\mathrm{CH}_{3}\right), 25.89$ $\left(3 \mathrm{CH}_{3}\right), 26.35\left(\mathrm{CH}_{3}\right), 30.24(\mathrm{C}), 89.43(\mathrm{CH}), 102.43$ $\left(\mathrm{CH}_{2}\right), 108.99(\mathrm{CH}), 110.14(\mathrm{CH}), 126.97(\mathrm{CH})$, $129.53(2 \mathrm{CH}), 129.98(\mathrm{CH}), 133.05(\mathrm{C}), 144.11(\mathrm{CH})$, 148.27 (C), $151.41(\mathrm{C}), 153.43(\mathrm{C}), 196.88(\mathrm{C}=\mathrm{O})$. MS (ESI, $m / z$ ): $385.1\left[\mathrm{M}^{+}+1\right]$. Anal. calcd for $\mathrm{C}_{22} \mathrm{H}_{28} \mathrm{O}_{4} \mathrm{Si}$ (384.18): C 68.71, H 7.32; found C 68.72, H 7.24.

$2.2 \mathrm{j}$ 1-Benzo[d][1,3]dioxol-5-yl)-2-(tertbutyldimethylsilyloxy)-2-(3-bromophenyl)ethanone (3j): Yield: 89\%, mp: $210-212^{\circ} \mathrm{C}$. IR $\left(\mathrm{KBr}, \mathrm{cm}^{-1}\right): 3347,3138,2242$, 1712, 1597, 1213, 867. ${ }^{1} \mathrm{H}$ NMR $\left(\mathrm{CDCl}_{3}\right) \delta: 0.25(6 \mathrm{H}$ s), $0.99(9 \mathrm{H}, \mathrm{s}), 5.97(1 \mathrm{H}, \mathrm{s}), 6.07(2 \mathrm{H}, \mathrm{s}), 7.11(1 \mathrm{H}$, $\mathrm{s}), 7.19(1 \mathrm{H}, \mathrm{d}, J=8.8 \mathrm{~Hz}), 7.32(1 \mathrm{H}, \mathrm{d}, J=6.4 \mathrm{~Hz})$, $7.46(1 \mathrm{H}, \mathrm{t}, J=8.8,7.6 \mathrm{~Hz}), 7.54(1 \mathrm{H}, \mathrm{d}, J=4.4 \mathrm{~Hz})$, $7.63(1 \mathrm{H}, \mathrm{d}, J=8.8 \mathrm{~Hz}), 7.79(1 \mathrm{H}, \mathrm{d}, J=8.4 \mathrm{~Hz})$. ${ }^{13} \mathrm{C}$ NMR $\left(\mathrm{CDCl}_{3}\right) \delta:-2.54\left(\mathrm{CH}_{3}\right), 25.85\left(3 \mathrm{CH}_{3}\right)$, $29.95(\mathrm{C}), 90.44(\mathrm{CH}), 103.77\left(\mathrm{CH}_{2}\right), 109.64(\mathrm{CH})$, $110.97(\mathrm{CH}), 127.15(\mathrm{CH}), 129.35(2 \mathrm{CH}), 129.74$ $(\mathrm{CH}), 132.81(\mathrm{C}), 143.41(\mathrm{CH}), 148.13(\mathrm{C}), 151.34$ (C), 153.87 (C), $196.97(\mathrm{C}=\mathrm{O})$. MS (ESI, $m / z)$ : 450.0 $\left[\mathrm{M}^{+}+1\right], 451.2\left[\mathrm{M}^{+}+2\right]$. Anal. calcd for $\mathrm{C}_{21} \mathrm{H}_{25} \mathrm{BrO}_{4} \mathrm{Si}$ (449.04): C 56.14, H 5.67; found C 56.07, H 5.12.

2.2k 1-Benzo[d][1,3]dioxol-5-yl)-2-(tertbutyldimethylsilyloxy)-2-(3-chlorophenyl)ethanone (3k): Yield: $85 \%$, mp: $118-122^{\circ} \mathrm{C}$. IR $\left(\mathrm{KBr}, \mathrm{cm}^{-1}\right): 3338,3122,2257$, 1710, 1601, 1214, 989. ${ }^{1} \mathrm{H}$ NMR (DMSO- $\left.d_{6}\right) \delta: 0.24$ $(6 \mathrm{H}, \mathrm{s}), 1.05(9 \mathrm{H}, \mathrm{s}), 5.99(1 \mathrm{H}, \mathrm{s}), 6.05(2 \mathrm{H}, \mathrm{s}), 7.10$ $(1 \mathrm{H}, \mathrm{s}), 7.14(1 \mathrm{H}, \mathrm{d}, J=2.8 \mathrm{~Hz}), 7.38(1 \mathrm{H}, \mathrm{d}, J=$ $6.0 \mathrm{~Hz}), 7.49(1 \mathrm{H}, \mathrm{t}, J=19.6 \mathrm{~Hz}), 7.53(1 \mathrm{H}, \mathrm{d}, J=$ $8.4 \mathrm{~Hz}), 7.67(1 \mathrm{H}, \mathrm{d}, J=4.8 \mathrm{~Hz}), 7.82(1 \mathrm{H}, \mathrm{d}, J=$ $8.8 \mathrm{~Hz}) \cdot{ }^{13} \mathrm{C}$ NMR (DMSO- $\left.d_{6}\right) \delta:-2.64\left(\mathrm{CH}_{3}\right), 25.99$ $\left(3 \mathrm{CH}_{3}\right), 29.84(\mathrm{C}), 90.64(\mathrm{CH}), 103.33\left(\mathrm{CH}_{2}\right), 108.69$ $(\mathrm{CH}), 110.92(\mathrm{CH}), 127.14(\mathrm{CH}), 129.54(2 \mathrm{CH})$, $129.73(\mathrm{CH}), 132.82(\mathrm{C}), 143.47(\mathrm{CH}), 148.23(\mathrm{C})$, 151.42 (C), 153.72 (C), $197.01(\mathrm{C}=\mathrm{O})$. MS (ESI, $m / z): 404.9\left[\mathrm{M}^{+}\right], 405.2\left[\mathrm{M}^{+}+1\right]$; Anal. calcd for 
$\mathrm{C}_{21} \mathrm{H}_{25} \mathrm{ClO}_{4} \mathrm{Si}$ (404.92): C 62.28, $\mathrm{H}$ 6.24, $\mathrm{Cl}$ 8.73; found $\mathrm{C} 62.27, \mathrm{H} 6.28, \mathrm{Cl} 8.73$.

2.21 1-Benzo[d][1,3]dioxol-5-yl)-2-(tertbutyldimethylsilyloxy)-2-(3-hydroxyphenyl)ethanone (3l): Yield: 84\%, mp: $174-175^{\circ} \mathrm{C}$. IR $\left(\mathrm{KBr}, \mathrm{cm}^{-1}\right): 3546,3342,3165$, $2240,1707,1589,1235 .{ }^{1} \mathrm{H}$ NMR $\left(\mathrm{CDCl}_{3}\right) \delta: 0.23(6 \mathrm{H}$, s), $0.99(9 \mathrm{H}, \mathrm{s}), 5.97(1 \mathrm{H}, \mathrm{s}), 6.07(2 \mathrm{H}, \mathrm{s}), 7.12(1 \mathrm{H}$, s), $7.25(1 \mathrm{H}, \mathrm{d}, J=6.4 \mathrm{~Hz}), 7.37(1 \mathrm{H}, \mathrm{d}, J=6.8 \mathrm{~Hz})$, $7.46(1 \mathrm{H}, \mathrm{t}, J=4.8 \mathrm{~Hz}), 7.58(1 \mathrm{H}, \mathrm{d}, J=7.6 \mathrm{~Hz})$, $7.74(1 \mathrm{H}, \mathrm{d}, J=8.8 \mathrm{~Hz}), 7.88(1 \mathrm{H}, \mathrm{d}, J=8.8 \mathrm{~Hz})$, $10.54(1 \mathrm{H}, \mathrm{s}) \mathrm{ppm} .{ }^{13} \mathrm{C} \mathrm{NMR}\left(\mathrm{CDCl}_{3}\right) \delta:-2.45\left(\mathrm{CH}_{3}\right)$, $25.89\left(3 \mathrm{CH}_{3}\right), 30.88(\mathrm{C}), 90.45(\mathrm{CH}), 103.53\left(\mathrm{CH}_{2}\right)$, $109.77(\mathrm{CH}), 111.03(\mathrm{CH}), 127.18(\mathrm{CH}), 129.54$ (2CH), $129.77(\mathrm{CH}), 132.59(\mathrm{C}), 143.53(\mathrm{CH}), 148.77$ (C), 150.99 (C), 153.44 (C), 196.57 (C=O). MS (ESI, $m / z): 387.2\left[\mathrm{M}^{+}+1\right]$. Anal. calcd for $\mathrm{C}_{21} \mathrm{H}_{25} \mathrm{O}_{5} \mathrm{Si}$ (386.17): C 65.26, H 6.77; found C 65.16, H 6.71.

$2.2 \mathrm{~m}$ 1,2-Bis-benzo[d][1,3]dioxol-5-yl)-2-(tertbutyldimethylsilyloxy)-ethanone (3m): Yield: 87\%, mp: $125-129^{\circ} \mathrm{C}$. IR $\left(\mathrm{KBr}, \mathrm{cm}^{-1}\right): 3343,3121,2250,1717$, 1607, 1217; ${ }^{1} \mathrm{H}$ NMR $\left(\mathrm{CDCl}_{3}\right) \delta: 0.21(6 \mathrm{H}, \mathrm{s}), 1.11$ $(9 \mathrm{H}, \mathrm{s}), 6.02(1 \mathrm{H}, \mathrm{s}), 6.09(4 \mathrm{H}, \mathrm{s}), 6.82(1 \mathrm{H}, \mathrm{t}, J=$ $2.8,3.2 \mathrm{~Hz}), 6.89(1 \mathrm{H}, \mathrm{d}, J=4.0 \mathrm{~Hz}), 6.99(1 \mathrm{H}, \mathrm{d}, J$ $=4.8 \mathrm{~Hz}), 7.19(1 \mathrm{H}, \mathrm{d}, J=6.8 \mathrm{~Hz}), 7.38(1 \mathrm{H}, \mathrm{d}, J$ $=8.4 \mathrm{~Hz}), 7.49(1 \mathrm{H}, \mathrm{s}) \cdot{ }^{13} \mathrm{C} \mathrm{NMR}\left(\mathrm{CDCl}_{3}\right) \delta:-2.48$ $\left(\mathrm{CH}_{3}\right), 25.94\left(3 \mathrm{CH}_{3}\right), 30.55(\mathrm{C}), 86.24(\mathrm{CH}), 102.33$ $\left(\mathrm{CH}_{2}\right), 102.99\left(\mathrm{CH}_{2}\right), 108.69(\mathrm{CH}), 110.92(\mathrm{CH})$, $116.34(\mathrm{CH}), 125.14(\mathrm{CH}), 126.54(2 \mathrm{CH}), 129.73(\mathrm{C})$, 132.82 (C), 143.47 (C), 148.23 (C), 151.42 (C), 153.72 (C), $194.22(\mathrm{C}=\mathrm{O})$. MS (ESI, $m / z): 415.5\left[\mathrm{M}^{+}+1\right]$. Anal. calcd for $\mathrm{C}_{22} \mathrm{H}_{26} \mathrm{O}_{6} \mathrm{Si}$ (414.55): C 63.73, H 6.32; found $\mathrm{C} 63.77, \mathrm{H} 6.33$.

2.2n 1-Benzo[d][1,3]dioxol-5-yl)-2-(tertbutyldimethylsilyloxy)-2-(quinolin-2-yl)ethanone (3n): Yield: 89\%, mp: $224-227^{\circ} \mathrm{C}$. IR $\left(\mathrm{KBr}, \mathrm{cm}^{-1}\right): 3352,3017,2243$, 1707, 1611, 1238. ${ }^{1} \mathrm{H}$ NMR $\left(\mathrm{CDCl}_{3}\right) \delta: 0.23(6 \mathrm{H}, \mathrm{s})$, $1.01(9 \mathrm{H}, \mathrm{s}), 6.01(1 \mathrm{H}, \mathrm{s}), 6.07(2 \mathrm{H}, \mathrm{s}), 7.19(1 \mathrm{H}, \mathrm{d}, J$ $=8.0 \mathrm{~Hz}), 7.24(1 \mathrm{H}, \mathrm{d}, J=8.4 \mathrm{~Hz}), 7.42(1 \mathrm{H}, \mathrm{s}), 7.45$ $(1 \mathrm{H}, \mathrm{d}, J=8.4 \mathrm{~Hz}), 7.62(1 \mathrm{H}, \mathrm{t}, J=6.4 \mathrm{~Hz}), 7.83$ $(1 \mathrm{H}, \mathrm{t}, J=6.0 \mathrm{~Hz}), 7.98(1 \mathrm{H}, \mathrm{d}, J=8.8 \mathrm{~Hz}), 8.14$ $(1 \mathrm{H}, \mathrm{d}, J=8.8 \mathrm{~Hz}), 8.38(1 \mathrm{H}, \mathrm{d}, J=12.4 \mathrm{~Hz}) \cdot{ }^{13} \mathrm{C}$ NMR $\left(\mathrm{CDCl}_{3}\right) \delta:-2.54\left(\mathrm{CH}_{3}\right), 25.84\left(3 \mathrm{CH}_{3}\right), 30.68$ (C), $90.84(\mathrm{CH}), 102.03\left(\mathrm{CH}_{2}\right), 109.77(\mathrm{CH}), 110.68$ $(\mathrm{CH}), 123.87(\mathrm{CH}), 125.44(\mathrm{CH}), 126.77(\mathrm{C}), 127.18$ $(\mathrm{CH}), 129.54(\mathrm{CH}), 130.17(\mathrm{CH}), 132.55(\mathrm{C}), 142.59$ (CH), 147.53 (C), 148.77 (C), 153.99 (C), $158.44(\mathrm{C})$, $194.55(\mathrm{C}=\mathrm{O})$. MS (ESI, $m / z): 422.1\left[\mathrm{M}^{+}+1\right]$. Anal. calcd for $\mathrm{C}_{24} \mathrm{H}_{27} \mathrm{NO}_{4} \mathrm{Si}$ (421.17): $\mathrm{C} 68.36, \mathrm{H}$ 6.47, N 3.33; found C 68.37, H 6.43, N 3.38. 2.2o 1-Benzo[d][1,3]dioxol-5-yl)-2-(tertbutyldimethylsilyloxy)-2-(5-methyl-1H-indol-3-yl)ethanone (3o): Yield: $89 \%$, mp: $224-227^{\circ} \mathrm{C}$. IR $\left(\mathrm{KBr}, \mathrm{cm}^{-1}\right): 3398$, 3312, 3018, 2249, 1717, 1608, 1547, 1285. ${ }^{1} \mathrm{H}$ NMR $\left(\mathrm{CDCl}_{3}\right) \delta: 0.22(6 \mathrm{H}, \mathrm{s}), 1.05(9 \mathrm{H}, \mathrm{s}), 2.58(3 \mathrm{H}, \mathrm{s})$, $4.87(1 \mathrm{H}, \mathrm{brs}), 5.99(1 \mathrm{H}, \mathrm{s}), 6.07(2 \mathrm{H}, \mathrm{s}), 7.15(1 \mathrm{H}, \mathrm{d}$, $J=8.4 \mathrm{~Hz}), 7.22(1 \mathrm{H}, \mathrm{s}), 7.31(1 \mathrm{H}, \mathrm{d}, J=8.8 \mathrm{~Hz})$, $7.37(1 \mathrm{H}, \mathrm{d}, J=8.4 \mathrm{~Hz}), 7.42(1 \mathrm{H}, \mathrm{d}, J=10.4 \mathrm{~Hz})$, $7.63(1 \mathrm{H}, \mathrm{s}), 7.82(1 \mathrm{H}, \mathrm{d}, J=8.4 \mathrm{~Hz}) .{ }^{13} \mathrm{C} \mathrm{NMR}$ $\left(\mathrm{CDCl}_{3}\right) \delta:-2.47\left(\mathrm{CH}_{3}\right), 22.54\left(\mathrm{CH}_{3}\right), 25.84\left(3 \mathrm{CH}_{3}\right)$, $30.37(\mathrm{C}), 90.44(\mathrm{CH}), 102.03\left(\mathrm{CH}_{2}\right), 108.11(\mathrm{CH})$, $110.75(\mathrm{CH}), 111.68(\mathrm{CH}), 112.87(\mathrm{C}), 122.87(\mathrm{CH})$, $123.44(\mathrm{CH}), 125.77(\mathrm{CH}), 127.18(\mathrm{C}), 129.54(\mathrm{C})$, 131.88 (C), 134.57 (C), 148.77 (C), 153.99 (C), 194.52 $(\mathrm{C}=\mathrm{O})$. MS $(\mathrm{ESI}, m / z): 424.1\left[\mathrm{M}^{+}+1\right]$. Anal. calcd for $\mathrm{C}_{24} \mathrm{H}_{29} \mathrm{NO}_{4} \mathrm{Si}$ (423.17): C 68.06, H 6.94, N 3.32; found C 68.07, H 6.37, N 3.35.

$2.2 \mathrm{p} \quad$ 1-Benzo[d][1,3]dioxol-5-yl)-2-(tertbutyldimethylsilyloxy)-2-(1-methyl-1H-indol-3-yl)ethanone (3p): Yield: $81 \%$, mp: $175-177^{\circ} \mathrm{C}$. IR $\left(\mathrm{KBr}, \mathrm{cm}^{-1}\right): 3308$, 3023, 2257, 1707, 1612, 1215. ${ }^{1} \mathrm{H}$ NMR $\left(\mathrm{CDCl}_{3}\right) \delta$ : $0.24(6 \mathrm{H}, \mathrm{s}), 0.99(9 \mathrm{H}, \mathrm{s}), 3.86(3 \mathrm{H}, \mathrm{s}), 6.01(1 \mathrm{H}, \mathrm{s})$, $6.07(2 \mathrm{H}, \mathrm{s}), 6.88(1 \mathrm{H}, \mathrm{s}), 7.12(1 \mathrm{H}, \mathrm{d}, J=8.8 \mathrm{~Hz})$, $7.22(1 \mathrm{H}, \mathrm{d}, J=8.4 \mathrm{~Hz}), 7.37(1 \mathrm{H}, \mathrm{d}, J=8.0 \mathrm{~Hz})$, $7.38(1 \mathrm{H}, \mathrm{d}, J=4.8 \mathrm{~Hz}), 7.39(1 \mathrm{H}, \mathrm{s}), 7.52(1 \mathrm{H}, \mathrm{d}$, $J=8.8 \mathrm{~Hz}) 7.58(1 \mathrm{H}, \mathrm{d}, J=8.0 \mathrm{~Hz}) \cdot{ }^{13} \mathrm{C} \mathrm{NMR}$ $\left(\mathrm{CDCl}_{3}\right)$ \&: $-2.46\left(\mathrm{CH}_{3}\right), 25.43\left(3 \mathrm{CH}_{3}\right), 29.99(\mathrm{C})$, $34.54\left(\mathrm{CH}_{3}\right), 90.67(\mathrm{CH}), 102.08\left(\mathrm{CH}_{2}\right), 108.11(\mathrm{CH})$, $109.45(\mathrm{CH}), 110.75(\mathrm{CH}), 111.63(\mathrm{CH}), 112.54(\mathrm{C})$, $122.87(\mathrm{CH}), 123.44(\mathrm{CH}), 125.77(\mathrm{CH}), 127.18(\mathrm{C})$, 131.55 (C), 141.54 (C), 148.72 (C), 153.28 (C), 194.77 $(\mathrm{C}=\mathrm{O})$. MS (ESI, $m / z): 424.2\left[\mathrm{M}^{+}+1\right]$. Anal. calcd for $\mathrm{C}_{24} \mathrm{H}_{29} \mathrm{NO}_{4} \mathrm{Si}$ (423.17): C 68.06, H 6.94, N 3.32; found C 68.07, H 6.37, N 3.35.

$2.2 q$ 1-Benzo[d][1,3]dioxol-5-yl)-2-(tertbutyldimethylsilyloxy)-2-(1-methyl-1H-benzo[d]imidazole-2-yl)ethanone $(3 q)$ : Yield: $83 \%, \mathrm{mp}: 189-191^{\circ} \mathrm{C}$. IR $(\mathrm{KBr}$, $\left.\mathrm{cm}^{-1}\right): 3272,3002,2258,1711,1604,1228 .{ }^{1} \mathrm{H}$ NMR $\left(\mathrm{CDCl}_{3}\right) \delta: 0.23(6 \mathrm{H}, \mathrm{s}), 1.01(9 \mathrm{H}, \mathrm{s}), 3.94(3 \mathrm{H}, \mathrm{s}), 6.01$ $(1 \mathrm{H}, \mathrm{s}), 6.07(2 \mathrm{H}, \mathrm{s}), 7.07(1 \mathrm{H}, \mathrm{d}, J=2.8 \mathrm{~Hz}), 7.12$ $(1 \mathrm{H}, \mathrm{s}), 7.24(1 \mathrm{H}, \mathrm{d}, J=8.4 \mathrm{~Hz}), 7.32(1 \mathrm{H}, \mathrm{d}, J=$ $6.4 \mathrm{~Hz}), 7.39(1 \mathrm{H}, \mathrm{d}, J=6.8 \mathrm{~Hz}), 7.41(1 \mathrm{H}, \mathrm{t}, J=8.8$, $7.6 \mathrm{~Hz}), 7.52(1 \mathrm{H}, \mathrm{t}, J=6.0 \mathrm{~Hz}) .{ }^{13} \mathrm{C} \mathrm{NMR}\left(\mathrm{CDCl}_{3}\right) \delta$ : $-2.46\left(\mathrm{CH}_{3}\right), 25.75\left(3 \mathrm{CH}_{3}\right), 30.11(\mathrm{C}), 33.31\left(\mathrm{CH}_{3}\right)$, $90.47(\mathrm{CH}), 102.08\left(\mathrm{CH}_{2}\right), 108.18(\mathrm{CH}), 109.55(\mathrm{CH})$, $110.44(\mathrm{CH}), 119.55(\mathrm{CH}), 122.54(\mathrm{CH}), 123.46$ (2CH), 131.88 (C), $135.66(\mathrm{C}), 142.05$ (C), 143.54 (C), $148.74(\mathrm{C}), 153.27$ (C), $194.44(\mathrm{C}=\mathrm{O})$. MS (ESI, $m / z): 425.21\left[\mathrm{M}^{+}+1\right]$. Anal. calcd for $\mathrm{C}_{23} \mathrm{H}_{28} \mathrm{~N}_{2} \mathrm{O}_{4} \mathrm{Si}$ 
(424.17): C 65.06, H 6.64, N 6.62; found C 65.07, H $6.68, \mathrm{~N} 6.65$.

2.2r 1-Benzo[d][1,3]dioxol-5-yl)-2-(tertbutyldimethylsilyloxy)-2-(1-acetyl-1H-indol-3-yl)ethanone (3r): Yield: $77 \%$, mp: $238-240^{\circ} \mathrm{C}$. IR $\left(\mathrm{KBr}, \mathrm{cm}^{-1}\right)$ : 3356, 3122, 2255, 1702, 1728, 1612, 1232. ${ }^{1} \mathrm{H}$ NMR (DMSO$\left.d_{6}\right) \delta: 0.26(6 \mathrm{H}, \mathrm{s}), 0.99(9 \mathrm{H}, \mathrm{s}), 4.08(3 \mathrm{H}, \mathrm{s}), 6.01$ $(1 \mathrm{H}, \mathrm{s}), 6.07(2 \mathrm{H}, \mathrm{s}), 6.94(1 \mathrm{H}, \mathrm{s}), 7.17(1 \mathrm{H}, \mathrm{d}, J=$ $8.4 \mathrm{~Hz}), 7.22(1 \mathrm{H}, \mathrm{s}), 7.28(1 \mathrm{H}, \mathrm{d}, J=8.4 \mathrm{~Hz}), 7.38$ $(1 \mathrm{H}, \mathrm{d}, J=5.6 \mathrm{~Hz}), 7.41(1 \mathrm{H}, \mathrm{t}, J=12.4 \mathrm{~Hz}), 7.43$ $(1 \mathrm{H}, \mathrm{t}, J=4.4,3.6 \mathrm{~Hz}), 7.54(1 \mathrm{H}, \mathrm{d}, J=8.4 \mathrm{~Hz}) \cdot{ }^{13} \mathrm{C}$ NMR (DMSO- $\left.d_{6}\right) \delta:-2.46\left(\mathrm{CH}_{3}\right), 24.87\left(\mathrm{CH}_{3}\right), 25.87$ $\left(3 \mathrm{CH}_{3}\right), 30.22(\mathrm{C}), 90.67(\mathrm{CH}), 102.07\left(\mathrm{CH}_{2}\right), 108.13$ $(\mathrm{CH}), 109.44(\mathrm{CH}), 110.76(\mathrm{CH}), 111.67(\mathrm{CH}), 112.34$ (C), $122.84(\mathrm{CH}), 123.24(\mathrm{CH}), 125.22(\mathrm{CH}), 127.13$ (C), 131.57 (C), 141.58 (C), 148.71 (C), 153.27 (C), $168.44(\mathrm{C}=\mathrm{O}), 194.55(\mathrm{C}=\mathrm{O})$. MS (ESI, $2 \mathrm{~m} / z)$ : 451.1 $\left[\mathrm{M}^{+}+1\right]$. Anal. calcd for $\mathrm{C}_{25} \mathrm{H}_{29} \mathrm{NO}_{5} \mathrm{Si}$ (451.42): $\mathrm{C}$ 66.46, H 6.44, N 3.12; found C 66.57, H 6.37, N 3.15.

2.2s 1-Benzo[d][1,3]dioxol-5-yl)-2-(tertbutyldimethylsilyloxy)-2-(4-(piperidin-1-yl)phnyl)ethanone (3s): Yield: $82 \%$, mp: $186-188^{\circ} \mathrm{C}$. IR $\left(\mathrm{KBr}, \mathrm{cm}^{-1}\right)$ : 3344 , 3027, 2244, 1712, 1604, 1229. ${ }^{1} \mathrm{H}$ NMR $\left(\mathrm{CDCl}_{3}\right) \delta$ : $0.23(6 \mathrm{H}, \mathrm{s}), 0.97(9 \mathrm{H}, \mathrm{s}), 1.53(6 \mathrm{H}, \mathrm{m}), 3.58(4 \mathrm{H}, \mathrm{d}$, $J=8.4 \mathrm{~Hz}), 6.01(1 \mathrm{H}, \mathrm{s}), 6.07(2 \mathrm{H}, \mathrm{s}), 7.04(1 \mathrm{H}, \mathrm{d}$, $J=4.4 \mathrm{~Hz}), 7.18(1 \mathrm{H}, \mathrm{s}), 7.24(1 \mathrm{H}, \mathrm{d}, J=4.8 \mathrm{~Hz})$, $7.33(1 \mathrm{H}, \mathrm{d}, J=2.4 \mathrm{~Hz}), 7.44(1 \mathrm{H}, \mathrm{d}, J=8.4 \mathrm{~Hz})$, $7.52(1 \mathrm{H}, \mathrm{d}, J=8.4 \mathrm{~Hz}), 7.64(1 \mathrm{H}, \mathrm{d}, J=4.4 \mathrm{~Hz})$, $7.86(1 \mathrm{H}, \mathrm{d}, J=8.8 \mathrm{~Hz}) .{ }^{13} \mathrm{C} \mathrm{NMR}\left(\mathrm{CDCl}_{3}\right) \delta:-2.46$ $\left(\mathrm{CH}_{3}\right), 24.22\left(\mathrm{CH}_{2}\right), 25.54\left(2 \mathrm{CH}_{2}\right), 25.99\left(3 \mathrm{CH}_{3}\right)$, $30.25(\mathrm{C}), 54.89\left(2 \mathrm{CH}_{2}\right), 90.44(\mathrm{CH}), 101.57\left(\mathrm{CH}_{2}\right)$, $109.77(\mathrm{CH}), 110.12(\mathrm{CH}), 113.55(2 \mathrm{CH}), 123.77$ $(\mathrm{CH}), 127.05(\mathrm{C}), 129.84(\mathrm{CH}), 132.47(\mathrm{C}), 148.25$ (C), 151.37 (C), 153.83 (C), 196.22 (C=O). MS (ESI, $m / z)$ : $454.2\left[\mathrm{M}^{+}+1\right]$. Anal. calcd for $\mathrm{C}_{26} \mathrm{H}_{35} \mathrm{NO}_{4} \mathrm{Si}$ (453.56): C 68.84, H 7.77, N 3.05; found C 68.82, H 7.72, N 3.09.

2.2t 1-Benzo[d][1,3]dioxol-5-yl)-2-(tertbutyldimethylsilyloxy)-2-(4-morpholinophenyl)ethanone (3t): Yield: $86 \%$, mp: $201-203^{\circ} \mathrm{C}$. IR $\left(\mathrm{KBr}, \mathrm{cm}^{-1}\right): 3425$, 3212, 3033, 2248, 1702, 1608, 1357, 1217. ${ }^{1} \mathrm{H}$ NMR $\left(\mathrm{CDCl}_{3}\right) \delta: 0.23\left(6 \mathrm{H}, \mathrm{s}, \mathrm{CH}_{3}\right), 0.97(9 \mathrm{H}, \mathrm{s}), 3.13-3.22$ $(4 \mathrm{H}, \mathrm{d}), 3.61(4 \mathrm{H}, \mathrm{d}), 6.01(1 \mathrm{H}, \mathrm{s}), 6.07(2 \mathrm{H}, \mathrm{s}), 6.88$ $(1 \mathrm{H}, \mathrm{d}, J=2.8 \mathrm{~Hz}), 6.92(1 \mathrm{H}, \mathrm{d}, J=4.8 \mathrm{~Hz}), 7.14$ $(1 \mathrm{H}, \mathrm{d}, J=8.0 \mathrm{~Hz}), 7.28(1 \mathrm{H}, \mathrm{d}, J=4.8 \mathrm{~Hz}), 7.32$ $(1 \mathrm{H}, \mathrm{d}, J=4.0 \mathrm{~Hz}), 7.51(1 \mathrm{H}, \mathrm{d}, J=8.4 \mathrm{~Hz}), 7.64$ $(1 \mathrm{H}, \mathrm{s}) \mathrm{ppm} .{ }^{13} \mathrm{C} \mathrm{NMR}\left(\mathrm{CDCl}_{3}\right) \delta:-2.39\left(\mathrm{CH}_{3}\right), 25.43$ $\left(3 \mathrm{CH}_{3}\right), 30.44(\mathrm{C}), 55.42\left(2 \mathrm{CH}_{2}\right), 68.34\left(2 \mathrm{CH}_{2}\right), 90.55$
$(\mathrm{CH}), 101.54\left(\mathrm{CH}_{2}\right), 108.55(\mathrm{CH}), 110.8(\mathrm{CH}), 114.26$ $(\mathrm{CH}), 125.11(\mathrm{CH}), 129.55(\mathrm{CH}), 130.84(\mathrm{C}), 132.47$ (CH), 147.99 (C), 148.86 (C), 153.88 (C), 196.94 $(\mathrm{C}=\mathrm{O})$. MS (ESI, $m / z): 456.0\left[\mathrm{M}^{+}+1\right]$. Anal. calcd for $\mathrm{C}_{25} \mathrm{H}_{33} \mathrm{NO}_{5} \mathrm{Si}$ (455.23): C 65.91, H 7.34, N 3.05; found C 65.91, H 7.32, N 3.01.

\subsection{General procedure for the synthesis of compounds $(\mathbf{4 a - t})$}

To a solution of 3 ( 1 eq.) in THF was added $10 \%$ $\mathrm{HCl}(25 \mathrm{~mL} / \mathrm{g}$ of starting material) at room temperature and stirring was continued for 16-28 h. The mixture was diluted with $\mathrm{H}_{2} \mathrm{O}$ and extracted with EtOAc. The organic extract was washed with brine dried over anhydrous $\mathrm{Na}_{2} \mathrm{SO}_{4}$ and the solvent was evaporated under reduced pressure. The residue obtained was purified by CC silica gel (60-120 mesh) EtOAc -petroleum ether (5:1) to give compound $\mathbf{4 a - 4 t}$ in $78-89 \%$ yield.

2.3a 1-(Benzo[d][1,3]dioxol-5-yl)-2-cyclohexyl-2hydroxyethanone $(4 \mathrm{e})$ : Yield: $88 \%$, mp: $231-232^{\circ} \mathrm{C}$. IR $\left(\mathrm{KBr} \mathrm{cm}{ }^{-1}\right)$ : 3508, 3307, 3034, 2254, 1708, 1607, $1120,947 .{ }^{1} \mathrm{H}$ NMR $\left(\mathrm{CDCl}_{3}\right): \delta=1.43-1.58(11 \mathrm{H}, \mathrm{m})$, $1.99(1 \mathrm{H}, \mathrm{m}), 4.98(1 \mathrm{H}, \mathrm{s}), 5.98(1 \mathrm{H}, \mathrm{brs}), 6.05(2 \mathrm{H}$, s), $6.94(1 \mathrm{H}, \mathrm{d}, J=4.4 \mathrm{~Hz}), 7.42(1 \mathrm{H}, \mathrm{s}), 7.58(1 \mathrm{H}, \mathrm{d}$, $J=8.8 \mathrm{~Hz}) \mathrm{ppm} .{ }^{13} \mathrm{C} \mathrm{NMR}\left(\mathrm{CDCl}_{3}\right) \delta: 25.88\left(2 \mathrm{CH}_{2}\right)$, $26.97\left(\mathrm{CH}_{2}\right), 27.08\left(2 \mathrm{CH}_{2}\right), 38.51(\mathrm{CH}), 86.61(\mathrm{CH})$, $102.34\left(\mathrm{CH}_{2}\right), 108.72(\mathrm{CH}), 111.46(\mathrm{CH}), 124.12(\mathrm{CH})$, 126.05 (C), 148.73 (C), 153.90 (C), $196.45(\mathrm{C}=\mathrm{O})$. MS (ESI, $m / z): 263.1\left[\mathrm{M}^{+}+1\right]$. Anal. calcd for $\mathrm{C}_{15} \mathrm{H}_{18} \mathrm{O}_{4}$ (262.34): C 68.64, H 6.93; found C 68.80, H 6.96.

\subsection{General procedure for the synthesis of compound $(5 a-t)$}

A mixture of compound 4 ( 1 eq.), $\mathrm{EDCl}$ (1 eq.) and DMAP (1 eq.) was stirred in dry $\mathrm{CH}_{2} \mathrm{Cl}_{2}$ at room temperature under $\mathrm{N}_{2}$ atmosphere. $\mathrm{N}$-acetyl-4piperidinecarboxylic acid (1 eq.) was added to the mixture and stirring was continued at room temperature for $18-37 \mathrm{~h}$. The mixture was diluted with $\mathrm{H}_{2} \mathrm{O}$ and extracted with $\mathrm{CH}_{2} \mathrm{Cl}_{2}$. The organic extract was washed with brine, dried over anhydrous $\mathrm{Na}_{2} \mathrm{SO}_{4}$ and concentrated under reduced pressure. The residue obtained was purified by $\mathrm{CC}$ [silica gel (60-120 mesh), MeOH/ $\left.\mathrm{CH}_{2} \mathrm{Cl}_{2}(1: 15)\right]$ to get 5a-t in 68-82\% yield.

2.4a 2-(Benzo[d][1,3]dioxol-5-yl)-2-oxo-1-cyclohexylethyl-1-acetylpiperidine-4-carboxylate (5e): Yield: 88\%, mp: $231-232^{\circ} \mathrm{C}$. IR (KBr, $\left.\mathrm{cm}^{-1}\right)$ : 3284, 3057, 
$1728,1712,1699,1600,1354,1219,1122,1054 .{ }^{1} \mathrm{H}$ NMR $\left(\mathrm{CDCl}_{3}\right) \delta: 1.26-1.34(10 \mathrm{H}, \mathrm{m}), 1.79-1.99(4 \mathrm{H}$, $\mathrm{t}, J=12.8,1.6 \mathrm{~Hz}), 2.36(3 \mathrm{H}, \mathrm{s}), 2.52(1 \mathrm{H}, \mathrm{m}),(1 \mathrm{H}$, s), $3.31(4 \mathrm{H}, \mathrm{m}), 5.36(1 \mathrm{H}, \mathrm{d}, J=2.8 \mathrm{~Hz}), 6.07(2 \mathrm{H}, \mathrm{s})$, $6.98(1 \mathrm{H}, \mathrm{d}, J=8.4 \mathrm{~Hz}), 7.45(1 \mathrm{H}, \mathrm{s}), 7.68(1 \mathrm{H}, \mathrm{d}, J=$ $8.8 \mathrm{~Hz}) \mathrm{ppm} .{ }^{13} \mathrm{C} \mathrm{NMR}\left(\mathrm{CDCl}_{3}\right) \delta: 22.99\left(\mathrm{CH}_{3}\right), 25.66$ $\left(2 \mathrm{CH}_{2}\right), 26.07\left(\mathrm{CH}_{2}\right), 27.16\left(2 \mathrm{CH}_{2}\right), 29.44\left(2 \mathrm{CH}_{2}\right)$, $34.55(\mathrm{CH}), 41.75(\mathrm{CH}), 45.89\left(2 \mathrm{CH}_{2}\right), 91.05(\mathrm{CH})$, $102.44\left(\mathrm{CH}_{2}\right), 107.77(\mathrm{CH}), 112.41(\mathrm{CH}), 124.34(\mathrm{CH})$, $126.15(\mathrm{C}), 149.01(\mathrm{C}), 153.84(\mathrm{C}), 172.55(\mathrm{C}=\mathrm{O})$, 174.26 (C=O), $196.43(\mathrm{C}=\mathrm{O})$. MS (ESI, $m / z): 410.1$ $\left[\mathrm{M}^{+}+1\right]$. Anal. calcd for $\mathrm{C}_{23} \mathrm{H}_{23} \mathrm{NO}_{6}$ (409.45): $\mathrm{C} 67.54$, H 5.63, N 3.45; found C 67.57, H 5.64, N 3.45.

\subsection{General procedure for the synthesis of compound $(6 a-t)$}

To a solution of 5 (10 eq.) in $\mathrm{AcOH}$ was added $\mathrm{NH}_{4} \mathrm{OAc}$ (20 eq.) at room temperature and mixture was heated to $90^{\circ} \mathrm{C}$ for $30 \mathrm{~h}$. The mixture was cooled to room temperature, quenched with saturated $\mathrm{NaHCO}_{3}$ solution and extracted with EtOAc. The organic extract was dried over anhydrous $\mathrm{Na}_{2} \mathrm{SO}_{4}$ and the solvent was evaporated under reduced pressure. The residue was purified by $\mathrm{CC}$ [silica gel (60-120 mesh) $\mathrm{MeOH} / \mathrm{CH}_{2} \mathrm{Cl}_{2}$; table 1)].

\section{5a 4-(Benzo[d][1,3]dioxol-5-yl)-5-(furon-3-yl)-2-}

(1-acetylpiperidin-4-yl)oxazole (6a): $\mathrm{Mp}: 124-126^{\circ} \mathrm{C}$. IR $\left(\mathrm{KBr}, \mathrm{cm}^{-1}\right): 3057,1712,1678,1652,1612,1353$, 1207, 1143, 1023, 974. ${ }^{1} \mathrm{H}$ NMR $\left(\mathrm{CDCl}_{3}\right) \delta: 1.52$ $(4 \mathrm{H}, \mathrm{t}), 2.38(3 \mathrm{H}, \mathrm{s}), 2.84(1 \mathrm{H}, \mathrm{m}), 3.25(4 \mathrm{H}, \mathrm{t}, J$ $=9.6 \mathrm{~Hz}), 6.08(2 \mathrm{H}, \mathrm{s}), 6.76(1 \mathrm{H}, \mathrm{d}, J=7.6 \mathrm{~Hz})$, $6.99(1 \mathrm{H}, \mathrm{d}, J=8.4 \mathrm{~Hz}), 7.13(1 \mathrm{H}, \mathrm{d}, J=12.8 \mathrm{~Hz})$, $7.21(1 \mathrm{H}, \mathrm{d}, J=8.0 \mathrm{~Hz}), 7.33(1 \mathrm{H}, \mathrm{s}), 7.78(1 \mathrm{H}$, s). ${ }^{13} \mathrm{C} \mathrm{NMR}\left(\mathrm{CDCl}_{3}\right) \delta: 22.57\left(\mathrm{CH}_{3}\right), 30.44\left(2 \mathrm{CH}_{2}\right)$, $38.44(\mathrm{CH}), 47.34\left(2 \mathrm{CH}_{2}\right), 101.51\left(\mathrm{CH}_{2}\right), 108.88$ $(\mathrm{CH}), 109.23(\mathrm{CH}), 110.41(\mathrm{CH}), 121.22(\mathrm{CH}), 129.32$ (C), $131.25(\mathrm{C}), 140.15(\mathrm{CH}), 146.43(\mathrm{CH}), 148.43$ (C), 149.09 (C), 150.21 (C), 171.34 (C), 175.36 $(\mathrm{C}=\mathrm{O})$. MS (ESI, $m / z)$ : $381.0\left[\mathrm{M}^{+}+1\right]$. Anal. calcd for $\mathrm{C}_{21} \mathrm{H}_{20} \mathrm{~N}_{2} \mathrm{O}_{5}$ (380.36): C 66.31, H 5.32, N 7.35; found $\mathrm{C}$ 66.37, H 5.34, N 7.24.

$2.5 \mathrm{~b} \quad 4-($ Benzo[d][1,3]dioxol-5-yl)-5-(furon-2-yl)-2(1-acetylpiperidin-4-yl)oxazole (6b): $\mathrm{Mp}: 147-149^{\circ} \mathrm{C}$. IR $\left(\mathrm{KBr}, \mathrm{cm}^{-1}\right)$ : 3105, 1717, 1656, 1641, 1603, 1341, $1212,1152,1011,955 .{ }^{1} \mathrm{H}$ NMR $\left(\mathrm{CDCl}_{3}\right) \delta:=1.54$ $(4 \mathrm{H}, \mathrm{t}, J=2.8,3.6 \mathrm{~Hz}), 2.42(3 \mathrm{H}, \mathrm{s}), 2.87(1 \mathrm{H}, \mathrm{m})$, $3.24(4 \mathrm{H}, \mathrm{t}, J=4.0,4.8 \mathrm{~Hz}), 6.09(2 \mathrm{H}, \mathrm{s}), 6.89(1 \mathrm{H}$, $\mathrm{d}, J=8.8 \mathrm{~Hz}), 6.93(1 \mathrm{H}, \mathrm{t}, J=19.6 \mathrm{~Hz}), 7.17(1 \mathrm{H}$, $\mathrm{d}, J=6.0 \mathrm{~Hz}), 7.28(1 \mathrm{H}, \mathrm{d}, J=4.8 \mathrm{~Hz}), 7.37(1 \mathrm{H}, \mathrm{s})$, $7.72(1 \mathrm{H}, \mathrm{s}) .{ }^{13} \mathrm{C}$ NMR $\left(\mathrm{CDCl}_{3}\right) \delta: 21.99\left(\mathrm{CH}_{3}\right), 29.41$ $\left(2 \mathrm{CH}_{2}\right), 38.47(\mathrm{CH}), 47.77\left(2 \mathrm{CH}_{2}\right), 102.08\left(\mathrm{CH}_{2}\right)$, $108.28(\mathrm{CH}), 109.45(\mathrm{CH}), 110.47(\mathrm{CH}), 122.27(\mathrm{CH})$, $129.11(\mathrm{C}), 131.35(\mathrm{C}), 139.71(\mathrm{CH}), 146.13(\mathrm{CH})$, 147.47 (C), 149.02 (C), 150.77 (C), 171.87 (C), 174.98 $(\mathrm{C}=\mathrm{O})$. MS (ESI, $m / z): 381.1\left[\mathrm{M}^{+}+1\right]$. Anal. calcd for $\mathrm{C}_{21} \mathrm{H}_{20} \mathrm{~N}_{2} \mathrm{O}_{5}$ (380.36): C 66.37, H 5.38, N 7.27; found C 66.37, H 5.38, N 7.22.

$2.5 \mathrm{c}$ 4-(Benzo[d][1,3]dioxol-5-yl)-5-(thiophen-2-yl)2-(1-acetylpiperidin-4-yl)oxazole (6c): $\mathrm{IR}\left(\mathrm{KBr}, \mathrm{cm}^{-1}\right)$ : 3112, 1708, 1646, 1627, 1600, 1354, 1223, 1153, 1087. ${ }^{1} \mathrm{H}$ NMR $\left(\mathrm{CDCl}_{3}\right) \delta: 1.53(4 \mathrm{H}, \mathrm{t}), 2.44(3 \mathrm{H}, \mathrm{s}), 2.82$ $(1 \mathrm{H}, \mathrm{m}), 3.24(4 \mathrm{H}, \mathrm{t}, J=2.2 \mathrm{~Hz}), 6.11(2 \mathrm{H}, \mathrm{s}), 6.82$ $(1 \mathrm{H}, \mathrm{d}, J=7.2 \mathrm{~Hz}), 6.96(1 \mathrm{H}, \mathrm{t}, J=8.4 \mathrm{~Hz}), 7.08(1 \mathrm{H}$, $\mathrm{d}, J=6.4 \mathrm{~Hz}), 7.17(1 \mathrm{H}, \mathrm{d}, J=8.8 \mathrm{~Hz}), 7.38(1 \mathrm{H}, \mathrm{s})$, $7.74(1 \mathrm{H}, \mathrm{s}) .{ }^{13} \mathrm{C} \mathrm{NMR}\left(\mathrm{CDCl}_{3}\right) \delta: 22.07\left(\mathrm{CH}_{3}\right), 29.88$ $\left(2 \mathrm{CH}_{2}\right), 37.55(\mathrm{CH}), 48.24\left(2 \mathrm{CH}_{2}\right), 102.11\left(\mathrm{CH}_{2}\right)$, $108.27(\mathrm{CH}), 109.46(\mathrm{CH}), 110.42(\mathrm{CH}), 122.24(\mathrm{CH})$, $128.23(\mathrm{C}), 130.39(\mathrm{C}), 139.77(\mathrm{CH}), 146.18(\mathrm{CH})$, 147.88 (C), 149.35 (C), 151.48 (C), 172.02 (C), 175.28 $(\mathrm{C}=\mathrm{O})$. MS (ESI, $m / z): 397.2\left[\mathrm{M}^{+}+1\right]$. Anal. calcd for $\mathrm{C}_{21} \mathrm{H}_{20} \mathrm{~N}_{2} \mathrm{O}_{4} \mathrm{~S}$ (396.38): C 63.67, H 5.09, N 7.08; found $\mathrm{C}$ 63.66, H 5.14, N 7.06.

2.5d 4-(Benzo[d][1,3]dioxol-5-yl)-5-(pyridin-4-yl)2-(1-acetylpiperidin-4-yl)oxazole (6d): Mp: 187$190^{\circ} \mathrm{C}$. IR (KBr, cm $\left.{ }^{-1}\right)$ : 3124, 1720, 1653, 1638, 1612, 1547, 1483, 1348, 1202, 1146, 1018, 921. ${ }^{1} \mathrm{H}$ NMR $\left(\mathrm{CDCl}_{3}\right): \delta=1.49(4 \mathrm{H}, \mathrm{t}, J=2.4,3.2 \mathrm{~Hz}), 2.41(3 \mathrm{H}$, s), $2.85(1 \mathrm{H}, \mathrm{m}), 3.18(4 \mathrm{H}, \mathrm{t}, J=2.0,1.6 \mathrm{~Hz}), 6.08$ $(2 \mathrm{H}, \mathrm{s}), 6.98(1 \mathrm{H}, \mathrm{d}, J=4.4 \mathrm{~Hz}), 7.14(1 \mathrm{H}, \mathrm{d}, J=$ $8.4 \mathrm{~Hz}), 7.34(1 \mathrm{H}, \mathrm{s}), 7.84(2 \mathrm{H}, \mathrm{d}, J=8.8 \mathrm{~Hz}), 8.62$ $(2 \mathrm{H}, \mathrm{d}, J=12.4 \mathrm{~Hz}) \cdot{ }^{13} \mathrm{C} \mathrm{NMR}\left(\mathrm{CDCl}_{3}\right) \delta: 21.88$ $\left(\mathrm{CH}_{3}\right), 29.13\left(2 \mathrm{CH}_{2}\right), 36.24(\mathrm{CH}), 47.76\left(2 \mathrm{CH}_{2}\right)$, $101.87\left(\mathrm{CH}_{2}\right), 108.44(\mathrm{CH}), 108.98(\mathrm{CH}), 119.87$ $(\mathrm{CH}), 122.55(\mathrm{CH}), 127.77(\mathrm{CH}), 128.47(\mathrm{C}), 138.44$ (C), 143.33 (C), 147.54 (C), 147.88 (C), 149.88 (2C), 150.24 (C), 151.42 (C), $171.22(\mathrm{C}), 173.34(\mathrm{C}=\mathrm{O})$. MS (ESI, $m / z$ ): $392.1\left[\mathrm{M}^{+}+1\right]$. Anal. calcd for $\mathrm{C}_{22} \mathrm{H}_{21}$ $\mathrm{N}_{3} \mathrm{O}_{4}$ (391.42): C 67.48, H 5.39, N 10.75; found $\mathrm{C}$ 67.60, H 5.44, N 10.76 .

2.5e 4-(Benzo[d][1,3]dioxol-5-yl)-5-cyclohexyl-2-(1acetylpiperidin-4-yl)oxazole (6e): IR $\left(\mathrm{KBr}, \mathrm{cm}^{-1}\right)$ : 3142, 1700, 1647, 1633, 1645, 1532, 1302, 1247, 1136, 1011. ${ }^{1} \mathrm{H}$ NMR $\left(\mathrm{CDCl}_{3}\right) \delta: 1.28(10 \mathrm{H}, \mathrm{m}), 1 .(4 \mathrm{H}$, $\mathrm{t}, J=4.0,4.8 \mathrm{~Hz}), 2.38(3 \mathrm{H}, \mathrm{s}), 2.75(1 \mathrm{H}, \mathrm{m}), 2.83$ $(1 \mathrm{H}, \mathrm{m}), 3.37(4 \mathrm{H}, \mathrm{m}), 6.05(2 \mathrm{H}, \mathrm{s}), 6.99(1 \mathrm{H}, \mathrm{d}, J=$ $8.4 \mathrm{~Hz}), 7.35(1 \mathrm{H}, \mathrm{s}), 7.48(1 \mathrm{H}, \mathrm{d}, J=8.4 \mathrm{~Hz}) \cdot{ }^{13} \mathrm{C}$ NMR $\left(\mathrm{CDCl}_{3}\right) \delta: 21.56\left(\mathrm{CH}_{3}\right), 25.44\left(2 \mathrm{CH}_{2}\right), 26.12$ $\left(\mathrm{CH}_{2}\right), 29.22\left(2 \mathrm{CH}_{2}\right), 33.56\left(2 \mathrm{CH}_{2}\right), 35.87(\mathrm{CH}), 44.55$ 
Table 1. Synthesis of novel multi-substituted oxazole derivatives.

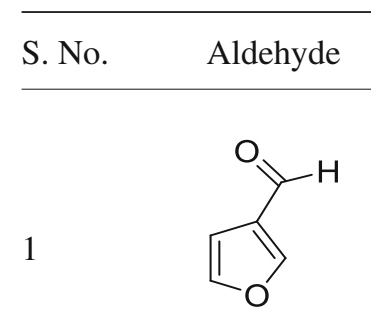

(a)
Product $^{\mathrm{a}}$<smiles>CC(=O)N1CCC(c2nc(-c3ccc4c(c3)OCO4)c(-c3ccoc3)o2)CC1</smiles><smiles>CC(=O)N1CCC(c2nc(-c3ccc4c(c3)OCO4)c(-c3ccco3)o2)CC1</smiles>

(c)<smiles>CC(=O)N1CCC(c2nc(-c3ccc4c(c3)OCO4)c(-c3cccs3)o2)CC1</smiles>

(b)<smiles>O=Cc1ccco1</smiles><smiles>O=Cc1cccs1</smiles>

\section{3}<smiles>O=Cc1ccncc1</smiles>

(d)<smiles>CC(=O)N1CCC(c2nc(-c3ccc4c(c3)OCO4)c(-c3ccncc3)o2)C(C(C)(F)F)C1</smiles>

5<smiles>O=CC1CCCCC1</smiles>

(e)<smiles>CC(=O)N1CCC(c2nc(-c3ccc4c(c3)OCO4)c(C3CCCCC3)o2)CC1</smiles><smiles>O=Cc1ccccc1</smiles>

(f)<smiles>CC(=O)N1CCC(c2nc(-c3ccc4c(c3)OCO4)c(-c3ccccc3)o2)CC1</smiles>

7<smiles>CC(=O)N1CCC(c2nc(-c3ccc4c(c3)OCO4)c(-c3cccc([N+](=O)[O-])c3)o2)CC1</smiles>

(g)<smiles>CC(=O)N1CCC(c2nc(-c3ccc4c(c3)OCO4)c(-c3ccccc3[N+](=O)[O-])o2)CC1</smiles><smiles>O=Cc1ccccc1[N+](=O)[O-]</smiles>

(h) 
Table 1. (continued)

S. No. Aldehyde

(i)<smiles>O=Cc1cccc(Br)c1</smiles>

(j)<smiles>O=Cc1cccc(Cl)c1</smiles>

(k)

11<smiles>CC(=O)N1CCC(c2nc(-c3ccc4c(c3)OCO4)c(-c3cccc(C)c3)o2)CC1</smiles>

(6j)<smiles>CC(=O)N1CCC(c2nc(-c3ccc4c(c3)OCO4)c(-c3cccc(Br)c3)o2)CC1</smiles>

$\mathrm{Cl}$<smiles>CC(=O)N1CCC(c2nc(-c3ccc4c(c3)OCO4)c(-c3cccc(Cl)c3)o2)CC1</smiles><smiles>O=Cc1cccc(O)c1</smiles>

(I)<smiles>O=Cc1cccc2c1OCO2</smiles>

(m)<smiles>CC(=O)N1CCC(c2nc(-c3ccc4c(c3)OCO4)c(-c3cccc(O)c3)o2)CC1</smiles><smiles>CC(=O)N1CCC(c2nc(-c3ccc4c(c3)OCO4)c(-c3ccc4c(c3)OCO4)o2)CC1</smiles>

13

14<smiles>O=Cc1ccc2ccccc2n1</smiles><smiles>CC(=O)N1CCC(c2nc(-c3ccc4c(c3)OCO4)c(-c3ccc4ccccc4n3)o2)CC1</smiles>

(n)

(6n)<smiles>Cc1ccc2[nH]cc(C=O)c2c1</smiles><smiles>CC(=O)N1CCC(c2nc(-c3ccc4c(c3)OCO4)c(-c3c[nH]c4ccc(C)cc34)o2)CC1</smiles> 
Table 1. (continued)

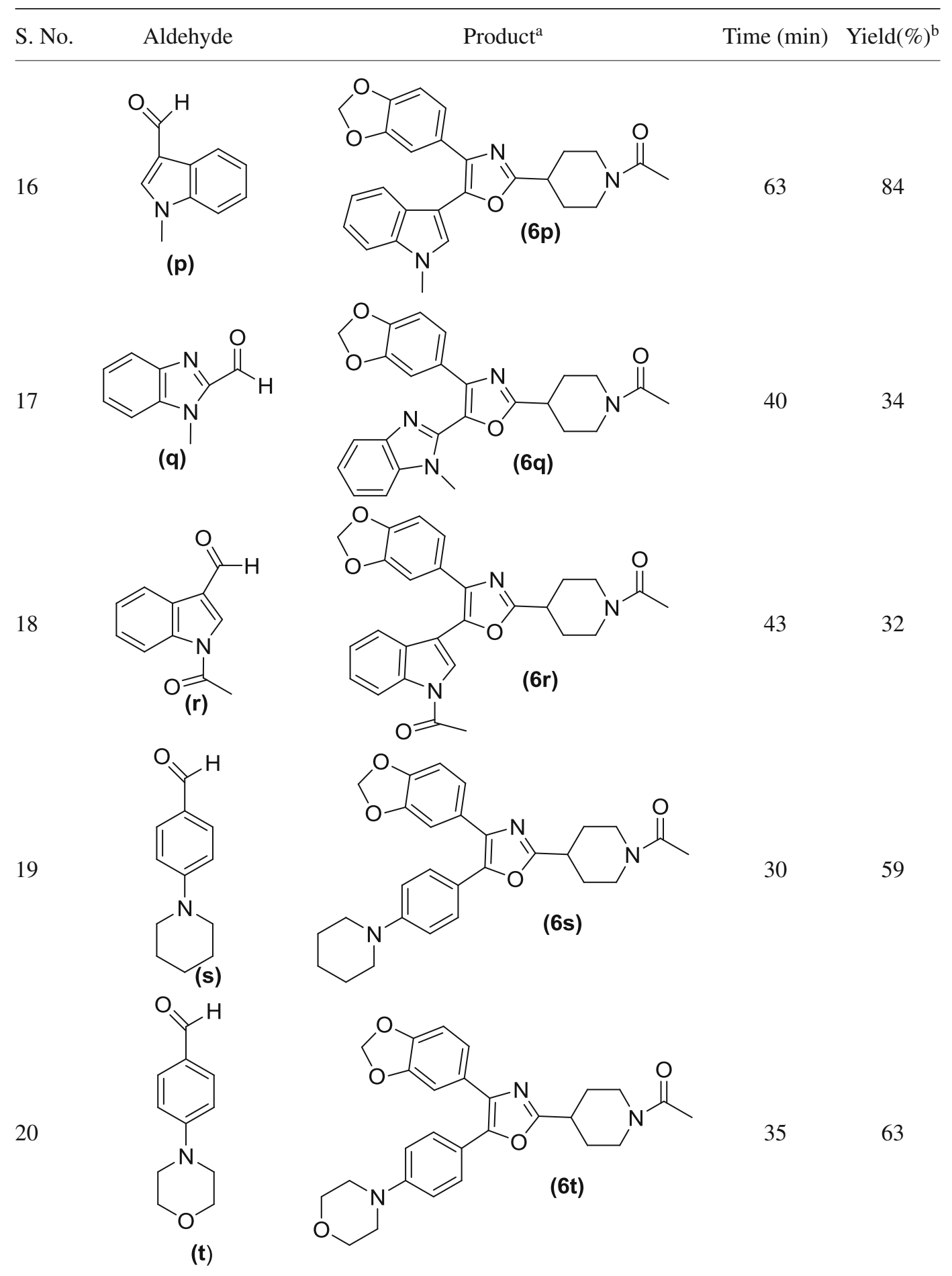

${ }^{\mathrm{a}}$ All products were characterized by elemental analysis IR, ${ }^{1} \mathrm{H}$ MR and ${ }^{13} \mathrm{C}$ NMR data

${ }^{b}$ Yield refers to pure products after purification by column chromatography

$\left(2 \mathrm{CH}_{2}\right), 47.75(\mathrm{CH}), 102.77\left(\mathrm{CH}_{2}\right), 107.54(\mathrm{CH})$ $108.56(\mathrm{CH}), 122.35(\mathrm{CH}), 125.65(\mathrm{C}), 126.34(\mathrm{C})$, 138.15 (C), 148.12 (C), 149.05 (C), 170.55 (C), 172.35 $(\mathrm{C}=\mathrm{O})$. MS (ESI, $m / z): 397.4\left[\mathrm{M}^{+}+1\right]$. Anal. calcd for $\mathrm{C}_{23} \mathrm{H}_{28} \mathrm{~N}_{2} \mathrm{O}_{4}$ (396.47): C 69.67, $\mathrm{H}$ 7.12, N 7.05; found $\mathrm{C} 69.59, \mathrm{H} 7.14, \mathrm{~N} 7.05$.
$2.5 f \quad 4-($ Benzo $[d][1,3]$ dioxol-5-yl)-5-phenyl-2-(1acetylpiperidin-4-yl)oxazole (6f): $\mathrm{IR}\left(\mathrm{KBr}, \mathrm{cm}^{-1}\right)$ : 3131, 1704, 1642, 1620, 1607, 1543, 1387, 1228, 1150, 1042. ${ }^{1} \mathrm{H}$ NMR $\left(\mathrm{CDCl}_{3}\right) \delta: 1.32(4 \mathrm{H}, \mathrm{t}, J=7.6 \mathrm{~Hz})$, $2.32(3 \mathrm{H}, \mathrm{s}), 2.80(1 \mathrm{H}, \mathrm{m}), 3.22(4 \mathrm{H}, \mathrm{t}, J=4.0 \mathrm{~Hz})$, $6.07(2 \mathrm{H}, \mathrm{s}), 6.99(1 \mathrm{H}, \mathrm{d}, J=6.0 \mathrm{~Hz}), 7.11(1 \mathrm{H}, \mathrm{d}, J$ 
$=8.4 \mathrm{~Hz}), 7.31(1 \mathrm{H}, \mathrm{s}), 7.44(1 \mathrm{H}, \mathrm{t}, J=8.0,8.8 \mathrm{~Hz})$, $7.55(2 \mathrm{H}, \mathrm{t}, J=6.4,3.2 \mathrm{~Hz}), 8.14(2 \mathrm{H}, \mathrm{d}, J=8.4 \mathrm{~Hz})$. ${ }^{13} \mathrm{C} \mathrm{NMR}\left(\mathrm{CDCl}_{3}\right) \delta: 21.22\left(\mathrm{CH}_{3}\right), 29.84\left(2 \mathrm{CH}_{2}\right), 35.35$ $(\mathrm{CH}), 45.84\left(2 \mathrm{CH}_{2}\right), 101.22\left(\mathrm{CH}_{2}\right), 108.44(\mathrm{CH})$, $109.56(\mathrm{CH}), 121.16(\mathrm{CH}), 126.36(\mathrm{C}), 127.44(2 \mathrm{CH})$, $128.09(\mathrm{C}), 128.88(\mathrm{CH}), 129.34(2 \mathrm{CH}), 137.22(\mathrm{C})$, 146.08 (C), 148.34 (C), 149.09 (C), 171.48 (C), 173.68 $(\mathrm{C}=\mathrm{O})$. MS (ESI, $m / z)$ : $391.0\left[\mathrm{M}^{+}+1\right]$. Anal. calcd for $\mathrm{C}_{23} \mathrm{H}_{22} \mathrm{~N}_{2} \mathrm{O}_{4}$ (390.34): C 70.74, $\mathrm{H}$ 5.69, $\mathrm{N}$ 7.21; found $\mathrm{C} 70.67, \mathrm{H}$ 5.72, N 7.26.

$2.5 \mathrm{~g}$ 4-(Benzo[d][1,3]dioxol-5-yl)-5-(3-nitrophenyl)2-(1-acetylpiperidin-4-yl)oxazole (6g): Mp: 243$245^{\circ} \mathrm{C}$. IR $\left(\mathrm{KBr}, \mathrm{cm}^{-1}\right): 3010,2254,1712,1608,1545$, 1322, 1207, 1155, 1002, 985. ${ }^{1} \mathrm{H}$ NMR $\left(\mathrm{CDCl}_{3}\right): \delta=$ $1.48(4 \mathrm{H}, \mathrm{t}, J=1.6,3.2 \mathrm{~Hz}), 2.37(3 \mathrm{H}, \mathrm{s}), 2.82(1 \mathrm{H}$, m), $3.23(4 \mathrm{H}, \mathrm{t}, J=2.8,9.6 \mathrm{~Hz}),, 6.09(2 \mathrm{H}, \mathrm{s}), 6.96$ $(1 \mathrm{H}, \mathrm{d}, J=8.4 \mathrm{~Hz}), 7.14(1 \mathrm{H}, \mathrm{d}, J=6.8 \mathrm{~Hz}), 7.33$ $(1 \mathrm{H}, \mathrm{s}), 7.82(1 \mathrm{H}, \mathrm{t}, J=6.4 \mathrm{~Hz}), 8.22(1 \mathrm{H}, \mathrm{d}, J=$ $8.8 \mathrm{~Hz}), 8.49(1 \mathrm{H}, \mathrm{d}, J=8.4 \mathrm{~Hz}), 8.65(1 \mathrm{H}, \mathrm{s}) .{ }^{13} \mathrm{C}$ NMR $\left(\mathrm{CDCl}_{3}\right) \delta: 21.85\left(\mathrm{CH}_{3}\right), 29.34\left(2 \mathrm{CH}_{2}\right), 34.34$ $(\mathrm{CH}), 44.87\left(2 \mathrm{CH}_{2}\right), 101.08\left(\mathrm{CH}_{2}\right), 108.22(\mathrm{CH})$, $109.02(\mathrm{CH}), 120.77(\mathrm{CH}), 123.44(\mathrm{CH}), 124.52(\mathrm{C})$, $127.09(\mathrm{C}), 129.99(\mathrm{CH}), 130.81(\mathrm{CH}), 132.56(\mathrm{C})$, 137.34 (C), 146.22 (C), 148.52 (C), 148.99 (C), 149.18 (C), 171.33 (C), $173.47(\mathrm{C}=\mathrm{O})$. MS (ESI, $m / z): 436.1$ $\left[\mathrm{M}^{+}+1\right]$. Anal. calcd for $\mathrm{C}_{23} \mathrm{H}_{21} \mathrm{~N}_{3} \mathrm{O}_{6}$ (435.12): $\mathrm{C}$ 63.44, H 4.89, N 9.64; found C 63.42, H 4.91, N 9.66.

2.5h 4-(Benzo[d][1,3]dioxol-5-yl)-5-(2-nitrophenyl)2-(1-acetylpiperidin-4-yl)oxazole (6h): $\mathrm{Mp}:>300^{\circ} \mathrm{C}$. IR $\left(\mathrm{KBr}, \mathrm{cm}^{-1}\right)$ : 3124, 3017, 2174, 1708, 1611, 1575, $1338,1242,1133,985,756 .{ }^{1} \mathrm{H}$ NMR (DMSO- $\left.d_{6}\right): \delta$ $=1.52(4 \mathrm{H}, \mathrm{t}, J=1.2,2.4 \mathrm{~Hz}), 2.39(3 \mathrm{H}, \mathrm{s}), 2.88(1 \mathrm{H}$, $\mathrm{m}), 3.42(4 \mathrm{H}, \mathrm{t}, J=4.4,4.8 \mathrm{~Hz}), 6.07(2 \mathrm{H}, \mathrm{s}), 6.99$ $(1 \mathrm{H}, \mathrm{d}, J=8.4 \mathrm{~Hz}), 7.11(1 \mathrm{H}, \mathrm{d}, J=8.0 \mathrm{~Hz}), 7.36$ $(1 \mathrm{H}, \mathrm{s}), 7.71(1 \mathrm{H}, \mathrm{t}, J=8.4,8.8 \mathrm{~Hz}), 7.85(1 \mathrm{H}, \mathrm{t}, J=$ $8.0,8.4 \mathrm{~Hz}), 8.13(1 \mathrm{H}, \mathrm{d}, J=6.4 \mathrm{~Hz}), 8.21(1 \mathrm{H}, \mathrm{d}, J$ $=8.8 \mathrm{~Hz}) \cdot{ }^{13} \mathrm{C}$ NMR $\left(\mathrm{DMSO}-d_{6}\right) \delta: 21.89\left(\mathrm{CH}_{3}\right), 29.37$ $\left(2 \mathrm{CH}_{2}\right), 34.33(\mathrm{CH}), 44.82\left(2 \mathrm{CH}_{2}\right), 101.12\left(\mathrm{CH}_{2}\right)$, $108.21(\mathrm{CH}), 109.01(\mathrm{CH}), 120.12(\mathrm{CH}), 123.22(\mathrm{CH})$, $124.56(\mathrm{C}), 127.29(\mathrm{C}), 129.76(\mathrm{CH}), 130.83(\mathrm{CH})$, 132.57 (C), 137.32 (C), 146.24 (C), 148.53 (C), 148.91 (C), 149.17 (C), 171.17 (C), $173.85(\mathrm{C}=\mathrm{O})$. MS (ESI, $m / z)$ : $436.1\left[\mathrm{M}^{+}+1\right]$. Anal. calcd for $\mathrm{C}_{23} \mathrm{H}_{21} \mathrm{~N}_{3} \mathrm{O}_{6}$ (435.12): C 63.45, H 4.87, N 9.62; found C 63.47, H 4.98, N 9.62.

$2.5 \mathrm{i} \quad 4-($ Benzo[d][1,3]dioxol-5-yl)-5-(m-tolyl)-2-(1acetylpiperidin-4-yl)oxazole (6i): Mp: $245-247^{\circ} \mathrm{C}$. IR $\left(\mathrm{KBr}, \mathrm{cm}^{-1}\right): 3254,3032,2161,1713,1619,1544$,
$1325,1254,1130,984,845 .{ }^{1} \mathrm{H}$ NMR $\left(\mathrm{CDCl}_{3}\right) \delta: 1.54$ $(4 \mathrm{H}, \mathrm{t}, J=3.6,4.0 \mathrm{~Hz}), 2.34(3 \mathrm{H}, \mathrm{s}), 2.42(3 \mathrm{H}, \mathrm{s}), 2.80$ $(1 \mathrm{H}, \mathrm{m}), 3.32-3.73(4 \mathrm{H}, \mathrm{t}, J=2.8,3.6 \mathrm{~Hz}), 6.09(2 \mathrm{H}$, s), $6.94(1 \mathrm{H}, \mathrm{d}, J=6.4 \mathrm{~Hz}), 7.08(1 \mathrm{H}, \mathrm{d}, J=8.4 \mathrm{~Hz})$, $7.24(1 \mathrm{H}, \mathrm{d}, J=8.0 \mathrm{~Hz}), 7.32(1 \mathrm{H}, \mathrm{s}), 7.48(1 \mathrm{H}, \mathrm{t}, J$ $=8.0,8.4 \mathrm{~Hz}), 7.74(1 \mathrm{H}, \mathrm{s}), 7.92(2 \mathrm{H}, \mathrm{d}, J=8.8 \mathrm{~Hz})$. ${ }^{13} \mathrm{C} \mathrm{NMR}\left(\mathrm{CDCl}_{3}\right) \delta: 21.22\left(\mathrm{CH}_{3}\right), 21.99\left(\mathrm{CH}_{3}\right), 29.74$ $\left(2 \mathrm{CH}_{2}\right), 35.44(\mathrm{CH}), 45.24\left(2 \mathrm{CH}_{2}\right), 101.20\left(\mathrm{CH}_{2}\right)$, $108.47(\mathrm{CH}), 109.74(\mathrm{CH}), 120.55(\mathrm{CH}), 122.68(\mathrm{CH})$, $126.42(\mathrm{C}), 129.23(\mathrm{CH}), 129.76(\mathrm{CH}), 131.24(\mathrm{C})$, 136.34 (C), 146.18 (C), 148.34 (C), 149.14 (C), 172.24 (C), $173.55(\mathrm{C}=\mathrm{O})$. MS (ESI, $m / z)$ : $404.3\left[\mathrm{M}^{+}\right], 405.1$ $\left[\mathrm{M}^{+}+1\right]$. Anal. calcd for $\mathrm{C}_{24} \mathrm{H}_{24} \mathrm{~N}_{2} \mathrm{O}_{4}$ (404.32): C 71.24, H 5.96, N 6.93; found C 71.27, H 5.92, N 6.93.

$2.5 \mathrm{j}$ 4-(Benzo[d][1,3]dioxol-5-yl)-5-(3-bromophenyl)2-(1-acetylpiperidin-4-yl)oxazole (6j): $\mathrm{Mp}: 198-199^{\circ} \mathrm{C}$. IR $\left(\mathrm{KBr}, \mathrm{cm}^{-1}\right)$ : 3147, 3008, 2036, 1725, 1654, 1562, $1308,1235,1131,1047,974 .{ }^{1} \mathrm{H}$ NMR $\left(\mathrm{CDCl}_{3}\right) \delta: 1.55$ $(4 \mathrm{H}, \mathrm{t}, J=2.8 \mathrm{~Hz}), 2.47(3 \mathrm{H}, \mathrm{s}), 2.82(1 \mathrm{H}, \mathrm{m}), 3.24$ $(4 \mathrm{H}, \mathrm{t}, J=6.4,6.8 \mathrm{~Hz}), 6.11(2 \mathrm{H}, \mathrm{s}), 6.98(1 \mathrm{H}, \mathrm{d}, J$ $=8.4 \mathrm{~Hz}), 7.11(1 \mathrm{H}, \mathrm{d}, J=8.4 \mathrm{~Hz}), 7.26(1 \mathrm{H}, \mathrm{s}), 7.41$ $(1 \mathrm{H}, \mathrm{t}, J=8.0,6.4 \mathrm{~Hz}), 7.44(1 \mathrm{H}, \mathrm{s}), 7.62(1 \mathrm{H}, \mathrm{d}, J=$ $4.8 \mathrm{~Hz}), 8.06(1 \mathrm{H}, \mathrm{d}, J=8.8 \mathrm{~Hz}) .{ }^{13} \mathrm{C} \mathrm{NMR}\left(\mathrm{CDCl}_{3}\right) \delta$ : $21.88\left(\mathrm{CH}_{3}\right), 29.33\left(2 \mathrm{CH}_{2}\right), 35.27(\mathrm{CH}), 45.74\left(2 \mathrm{CH}_{2}\right)$, $101.08\left(\mathrm{CH}_{2}\right), 108.12(\mathrm{CH}), 109.73(\mathrm{CH}), 120.75(\mathrm{CH})$, $122.62(\mathrm{CH}), 126.44(\mathrm{C}), 129.22(\mathrm{CH}), 129.44(\mathrm{CH})$, 131.22 (C), 136.55 (C), 146.17 (C), 148.33 (C), 149.17 (C), $172.21(\mathrm{C}), 173.57(\mathrm{C}=\mathrm{O})$. MS (ESI, $m / z): 469.2$ $\left[\mathrm{M}^{+}\right], 471.1\left[\mathrm{M}^{+}+2\right]$. Anal. calcd for $\mathrm{C}_{23} \mathrm{H}_{21} \mathrm{BrN}_{2} \mathrm{O}_{4}$ (469.22): C 58.87, H 4.54, N 5.96; found C 58.87, H 4.42, N 5.98 .

$2.5 \mathrm{k}$ 4-(Benzo[d][1,3]dioxol-5-yl)-5-(3-chlorophenyl)2-(1-acetylpiperidin-4-yl)oxazole (6k): Mp: $153-155^{\circ} \mathrm{C}$. IR $\left(\mathrm{KBr}, \mathrm{cm}^{-1}\right): 3138,3095,2022,1764,1624,1575$, 1311, 1233, 1137, 1045, 909, 856. ${ }^{1} \mathrm{H}$ NMR $\left(\mathrm{CDCl}_{3}\right)$ $\delta: 1.52(4 \mathrm{H}, \mathrm{t}, J=3.6,1.6 \mathrm{~Hz}), 2.41(3 \mathrm{H}, \mathrm{s}), 2.83(1 \mathrm{H}$, m), $3.24(4 \mathrm{H}, \mathrm{t}, J=2.8,2.4 \mathrm{~Hz}), 6.07(2 \mathrm{H}, \mathrm{s}), 6.97(1 \mathrm{H}$, $\mathrm{d}, J=8.4 \mathrm{~Hz}), 7.14(1 \mathrm{H}, \mathrm{d}, J=8.4 \mathrm{~Hz}), 7.27(1 \mathrm{H}, \mathrm{s})$, $7.45(1 \mathrm{H}, \mathrm{t}, J=8.4,7.6 \mathrm{~Hz}), 7.58(1 \mathrm{H}, \mathrm{s}), 7.72(1 \mathrm{H}$, $\mathrm{d}, J=8.0 \mathrm{~Hz}), 7.89(1 \mathrm{H}, \mathrm{d}, J=8.8 \mathrm{~Hz}) \cdot{ }^{13} \mathrm{C} \mathrm{NMR}$

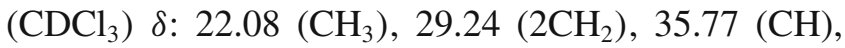
$45.22\left(2 \mathrm{CH}_{2}\right), 101.08\left(\mathrm{CH}_{2}\right), 108.14(\mathrm{CH}), 109.54$ $(\mathrm{CH}), 121.34(\mathrm{CH}), 123.52(\mathrm{CH}), 127.74(\mathrm{C}), 129.24$ $(\mathrm{CH}), 129.44(\mathrm{CH}), 132.54(\mathrm{C}), 136.74(\mathrm{C}), 145.23(\mathrm{C})$, 148.38 (C), 149.17 (C), $172.22(\mathrm{C}), 173.55(\mathrm{C}=\mathrm{O})$. MS (ESI, $m / z): 424.2\left[\mathrm{M}^{+}\right], 425.1\left[\mathrm{M}^{+}+1\right]$. Anal. calcd for $\mathrm{C}_{23} \mathrm{H}_{21} \mathrm{ClN}_{2} \mathrm{O}_{4}$ (424.22): C 65.08, $\mathrm{H}$ 4.96, $\mathrm{Cl}$ 8.35, N 6.56; found C 65.07, H 4.91, Cl 8.35, N 6.68. 
2.51 4-(Benzo[d][1,3]dioxol-5-yl)-5-(3-hydroxyphenyl)2-(1-acetylpiperidin-4-yl)oxazole (6l): Mp: $178-179^{\circ} \mathrm{C}$. IR $\left(\mathrm{KBr}, \mathrm{cm}^{-1}\right)$ : 3542, 3328, 3077, 2152, 1709, 1645, $1531,1344,1220,1187,1045 .{ }^{1} \mathrm{H}$ NMR $\left(\mathrm{CDCl}_{3}\right) \delta$ : $1.53(4 \mathrm{H}, \mathrm{t}, J=3.2,3.6 \mathrm{~Hz}), 2.44(3 \mathrm{H}, \mathrm{s}), 2.80(1 \mathrm{H}$, $\mathrm{m}), 3.24(4 \mathrm{H}, \mathrm{t}, J=2.8,3.6 \mathrm{~Hz}), 6.01(2 \mathrm{H}, \mathrm{s}), 6.98$ $(1 \mathrm{H}, \mathrm{d}, J=8.4 \mathrm{~Hz}), 7.11(1 \mathrm{H}, \mathrm{d}, J=6.0 \mathrm{~Hz}), 7.29$ $(1 \mathrm{H}, \mathrm{s}), 7.44(1 \mathrm{H}, \mathrm{t}, J=6.8,12.4 \mathrm{~Hz}), 7.59(1 \mathrm{H}, \mathrm{s})$, $7.77(1 \mathrm{H}, \mathrm{d}, J=8.0 \mathrm{~Hz}), 7.92(1 \mathrm{H}, \mathrm{d}, J=8.4 \mathrm{~Hz}) 8.64$ $(1 \mathrm{H}$, brs $) .{ }^{13} \mathrm{C} \mathrm{NMR}\left(\mathrm{CDCl}_{3}\right) \delta: 22.07\left(\mathrm{CH}_{3}\right), 29.24$ $\left(2 \mathrm{CH}_{2}\right), 35.77(\mathrm{CH}), 45.22\left(2 \mathrm{CH}_{2}\right), 101.11\left(\mathrm{CH}_{2}\right)$, 108.25 (CH), $110.24(\mathrm{CH}), 121.56(\mathrm{CH}), 123.53(\mathrm{CH})$, $128.08(\mathrm{C}), 129.09(\mathrm{CH}), 129.87(\mathrm{CH}), 131.24(\mathrm{C})$, 136.75 (C), 145.33 (C), 148.37 (C), 149.22 (C), 172.74 (C), $173.01(\mathrm{C}=\mathrm{O})$. MS (ESI, $m / z)$ : $407.2\left[\mathrm{M}^{+}+1\right]$. Anal. calcd for $\mathrm{C}_{23} \mathrm{H}_{22} \mathrm{~N}_{2} \mathrm{O}_{5}$ (406.23): C 67.96, H 5.46, N 6.88; found C 67.97, H 4.51, N 6.89.

$2.5 \mathrm{~m} \quad$ (4,5-di(Benzo[d][1,3]dioxol-5-yl)-2-(1-acetylpiperidin-4-yl)oxazole $(\mathbf{6 m})$ : $\mathrm{Mp}: 108-110^{\circ} \mathrm{C}$. IR $\left(\mathrm{KBr}, \mathrm{cm}^{-1}\right): 3144,3063,2147,1707,1600,1570$, 1307, 1234, 1131, 1009. ${ }^{1} \mathrm{H}$ NMR $\left(\mathrm{CDCl}_{3}\right) \delta: 1.35$ $(4 \mathrm{H}, \mathrm{t}, J=3.6,1.6 \mathrm{~Hz}), 2.39(3 \mathrm{H}, \mathrm{s}), 2.82(1 \mathrm{H}, \mathrm{m})$, $3.25(4 \mathrm{H}, \mathrm{t}, J=2.8,3.2 \mathrm{~Hz}), 6.07(2 \mathrm{H}, \mathrm{s}), 6.18(2 \mathrm{H}$, s), $6.94(2 \mathrm{H}, \mathrm{d}, J=8.8 \mathrm{~Hz}), 7.12(2 \mathrm{H}, \mathrm{d}, J=8.4 \mathrm{~Hz})$, $7.22(1 \mathrm{H}, \mathrm{s}), 7.43(1 \mathrm{H}, \mathrm{t}, J=8.8 \mathrm{~Hz}), 7.65(1 \mathrm{H}, \mathrm{d}, J=$ $8.4 \mathrm{~Hz}), 7.28(1 \mathrm{H}, \mathrm{d}, J=8.4 \mathrm{~Hz}) \cdot{ }^{13} \mathrm{C} \mathrm{NMR}\left(\mathrm{CDCl}_{3}\right) \delta$ : $22.14\left(\mathrm{CH}_{3}\right), 29.55\left(2 \mathrm{CH}_{2}\right), 35.32(\mathrm{CH}), 44.76\left(2 \mathrm{CH}_{2}\right)$, $101.08\left(\mathrm{CH}_{2}\right), 102.84\left(\mathrm{CH}_{2}\right), 108.89(2 \mathrm{CH}), 111.38$ $(2 \mathrm{CH}), 122.36(2 \mathrm{CH}), 128.28(\mathrm{C}), 129.87(\mathrm{CH}), 131.44$ (C), 136.64 (C), 142.68 (C), 148.36 (2C), 149.34 (2C), $172.55(\mathrm{C}), 173.85(\mathrm{C}=\mathrm{O})$. MS (ESI, $m / z)$ : 435.1 $\left[\mathrm{M}^{+}+1\right]$. Anal. calcd for $\mathrm{C}_{24} \mathrm{H}_{22} \mathrm{~N}_{2} \mathrm{O}_{6}$ (434.15): $\mathrm{C}$ 66.36, H 5.13, N 6.46; found C 66.37, H 5.11, N 6.39.

2.5n 4-(Benzo[d][1,3]dioxol-5-yl)-5-(quinolin-2-yl)2-(1-acetylpiperidin-4-yl)oxazole (6n): Mp: 223$224^{\circ} \mathrm{C}$. IR $\left(\mathrm{KBr}, \mathrm{cm}^{-1}\right): 3185,3005,2053,1715,1638$, 1574, 1328, 1222, 1154, 1024. ${ }^{1} \mathrm{H}$ NMR $\left(\mathrm{CDCl}_{3}\right) \delta$ : $1.34(4 \mathrm{H}, \mathrm{t}, J=2.4,1.6 \mathrm{~Hz}), 2.43(3 \mathrm{H}, \mathrm{s}), 2.82(1 \mathrm{H}$, m), $3.22(4 \mathrm{H}, \mathrm{t}, J=2.4,2.8 \mathrm{~Hz}), 6.04(2 \mathrm{H}, \mathrm{s}), 6.96$ $(1 \mathrm{H}, \mathrm{d}, J=8.4 \mathrm{~Hz}), 7.11(1 \mathrm{H}, \mathrm{d}, J=6.8 \mathrm{~Hz}), 7.24$ $(1 \mathrm{H}, \mathrm{s}), 7.48(1 \mathrm{H}, \mathrm{d}, J=6.0 \mathrm{~Hz}), 7.66(1 \mathrm{H}, \mathrm{t}, J=8.4$, $8.0 \mathrm{~Hz}), 7.81(2 \mathrm{H}, \mathrm{t}, J=6.4,7.6 \mathrm{~Hz}), 7.98(1 \mathrm{H}, \mathrm{d}, J$ $=8.8 \mathrm{~Hz}), 8.12(1 \mathrm{H}, \mathrm{d}, J=10.4 \mathrm{~Hz}), 8.46(2 \mathrm{H}, \mathrm{d}, J$ $=8.8 \mathrm{~Hz}) \cdot{ }^{13} \mathrm{C} \mathrm{NMR}\left(\mathrm{CDCl}_{3}\right) \delta: 21.25\left(\mathrm{CH}_{3}\right), 29.84$ $\left(2 \mathrm{CH}_{2}\right), 35.44(\mathrm{CH}), 46.87\left(2 \mathrm{CH}_{2}\right), 101.07\left(\mathrm{CH}_{2}\right)$, $108.11(\mathrm{CH}), 108.99(\mathrm{CH}), 119.22(\mathrm{CH}), 120.22(\mathrm{CH})$, $122.61(\mathrm{CH}), 125.42(\mathrm{C}), 126.24(\mathrm{C}), 127.46(\mathrm{CH})$, $128.47(\mathrm{CH}), 129.48(\mathrm{CH}), 137.42(\mathrm{C}), 138.76(\mathrm{CH})$, 139.48 (C), 145.22 (C), 148.46 (C), 149.847 (C), 157.24 (C), $171.42(\mathrm{C}), 173.39(\mathrm{C}=\mathrm{O})$. MS (ESI, $m / z)$ :
442.1 $\left[\mathrm{M}^{+}+1\right]$. Anal. calcd for $\mathrm{C}_{26} \mathrm{H}_{23} \mathrm{~N}_{3} \mathrm{O}_{4}$ (441.47): C 70.72, H 5.29, N 9.55; found C 70.67, H 5.34, N 9.56.

2.5o 4-(Benzo[d][1,3]dioxol-5-yl)-5-(5-methyl-1Hindol-3-yl)-2-(1-acetylpiperidin-4-yl)oxazole (6o): Mp: $192-195^{\circ} \mathrm{C}$. IR $\left(\mathrm{KBr}, \mathrm{cm}^{-1}\right): 3343,3012,2245,2146$, $1874,1719,1608,1547,1335,1225,1104,1034 .{ }^{1} \mathrm{H}$ $\operatorname{NMR}\left(\mathrm{CDCl}_{3}\right) \delta: 1.54(4 \mathrm{H}, \mathrm{t}, J=2.4,1.6 \mathrm{~Hz}), 2.41$ $(3 \mathrm{H}, \mathrm{s}), 2.52(3 \mathrm{H}, \mathrm{s}), 2.84(1 \mathrm{H}, \mathrm{m}), 3.42(4 \mathrm{H}, \mathrm{t}, J=$ $2.8,3.2 \mathrm{~Hz}), 6.08(2 \mathrm{H}, \mathrm{s}), 6.99(1 \mathrm{H}, \mathrm{d}, J=6.4 \mathrm{~Hz})$, $7.14(2 \mathrm{H}, \mathrm{d}, J=8.4 \mathrm{~Hz}), 7.33(1 \mathrm{H}, \mathrm{s}), 7.46(1 \mathrm{H}, \mathrm{d}$, $J=6.4 \mathrm{~Hz}), 7.89(1 \mathrm{H}, \mathrm{s}), 8.65(1 \mathrm{H}, \mathrm{s}), 10.34(1 \mathrm{H}$, brs). ${ }^{13} \mathrm{C} \mathrm{NMR}\left(\mathrm{CDCl}_{3}\right) \delta: 21.32\left(\mathrm{CH}_{3}\right), 22.02\left(\mathrm{CH}_{3}\right)$, $29.47\left(2 \mathrm{CH}_{2}\right), 34.65(\mathrm{CH}), 44.84\left(2 \mathrm{CH}_{2}\right), 99.87(\mathrm{C})$, $101.08\left(\mathrm{CH}_{2}\right), 108.64(\mathrm{CH}), 109.85(\mathrm{CH}), 112.36$ $(\mathrm{CH}), 121.56(\mathrm{CH}), 122.31(\mathrm{CH}), 123.44(\mathrm{CH}), 126.23$ (C), 127.59 (CH), $129.21(\mathrm{C}), 130.46(\mathrm{C}), 134.77$ (C), 136.42 (C), 146.62 (C), 148.95 (C), 149.12 (C), 170.49 (C), $172.55(\mathrm{C}=\mathrm{O})$. MS (ESI, $m / z)$ : $444.2\left[\mathrm{M}^{+}+1\right]$. Anal. calcd for $\mathrm{C}_{26} \mathrm{H}_{25} \mathrm{~N}_{3} \mathrm{O}_{4}$ (443.48): C 70.41, H 5.67, N 9.44; found C 70.43, H 5.68, N 9.42.

2.5p 4-(Benzo[d][1,3]dioxol-5-yl)-5-(1-methyl-1Hindol-3-yl)-2-(1-acetylpiperidin-4-yl)oxazole (6p): Mp: 245-247 ${ }^{\circ} \mathrm{C}$. IR $\left(\mathrm{KBr}, \mathrm{cm}^{-1}\right)$ : 3085, 2232, 2141, 1872, 1714, 1603, 1546, 1324, 1232, 1114. ${ }^{1} \mathrm{H} \mathrm{NMR}\left(\mathrm{CDCl}_{3}\right)$ $\delta: 1.52(4 \mathrm{H}, \mathrm{t}, J=3.6,1.6 \mathrm{~Hz}), 2.43(3 \mathrm{H}, \mathrm{s}), 2.84(1 \mathrm{H}$, $\mathrm{m}), 3.24(4 \mathrm{H}, \mathrm{t}, J=6.4,6.8 \mathrm{~Hz}), 3.76(3 \mathrm{H}, \mathrm{s}), 6.07$ $(2 \mathrm{H}, \mathrm{s}), 6.96(1 \mathrm{H}, \mathrm{d}, J=7.6 \mathrm{~Hz}), 7.11(2 \mathrm{H}, \mathrm{d}, J=$ $6.8 \mathrm{H}), 7.28(1 \mathrm{H}, \mathrm{s}), 7.48(2 \mathrm{H}, \mathrm{t}, J=8.0,7.6 \mathrm{~Hz}), 7.69$ $(1 \mathrm{H}, \mathrm{d}, J=8.4 \mathrm{~Hz}), 8.25(1 \mathrm{H}, \mathrm{d}, J=8.8 \mathrm{~Hz}) \cdot{ }^{13} \mathrm{C}$ NMR $\left(\mathrm{CDCl}_{3}\right) \delta: 21.87\left(\mathrm{CH}_{3}\right), 29.47\left(2 \mathrm{CH}_{2}\right), 34.65$ $(\mathrm{CH}), 35.97\left(\mathrm{CH}_{3}\right), 44.24\left(2 \mathrm{CH}_{2}\right), 99.86(\mathrm{C}), 101.11$ $\left(\mathrm{CH}_{2}\right), 108.84(\mathrm{CH}), 109.24(\mathrm{CH}), 110.31(\mathrm{CH}), 120.45$ $(\mathrm{CH}), 121.38(\mathrm{CH}), 122.64(\mathrm{CH}), 123.80(\mathrm{CH}), 126.08$ (C), $128.11(\mathrm{C}), 129.56(\mathrm{CH}), 136.23(\mathrm{C}), 137.46(\mathrm{C})$, 146.33 (C), 148.47 (C), 149.36 (C), 170.49 (C), 172.64 $(\mathrm{C}=\mathrm{O})$. MS (ESI, $m / z): 444.2\left[\mathrm{M}^{+}+1\right]$. Anal. calcd for $\mathrm{C}_{26} \mathrm{H}_{25} \mathrm{~N}_{3} \mathrm{O}_{4}$ (443.48): C 70.41, H 5.67, N 9.44; found C 70.43, H 5.68, N 9.42.

$2.5 \mathrm{q}$ 4-(Benzo[d][1,3]dioxol-5-yl)-5-(1-methyl-1Himidazol-2-yl)-2-(1-acetylpiperidin-4-yl)oxazole (6q): $\mathrm{Mp}:>300^{\circ} \mathrm{C}$. IR $\left(\mathrm{KBr}, \mathrm{cm}^{-1}\right): 3055,2267,2136,1804$, 1708, 1611, 1534, 1397, 1222, 1117. ${ }^{1} \mathrm{H} \mathrm{NMR}\left(\mathrm{CDCl}_{3}\right)$ $\delta: 1.35(4 \mathrm{H}, \mathrm{t}, J=2.0,1.2 \mathrm{~Hz}), 2.43(3 \mathrm{H}, \mathrm{s}), 2.82(1 \mathrm{H}$, m), $3.29(4 \mathrm{H}, \mathrm{t}, J=4.4,4.8 \mathrm{~Hz}), 4.08(3 \mathrm{H}, \mathrm{s}), 6.08(2 \mathrm{H}$, s), $6.99(1 \mathrm{H}, \mathrm{d}, J=6.0 \mathrm{~Hz}), 7.07(1 \mathrm{H}, \mathrm{d}, J=6.8 \mathrm{~Hz})$, $7.22(1 \mathrm{H}, \mathrm{t}, J=8.0,8.4 \mathrm{~Hz}), 7.63(1 \mathrm{H}, \mathrm{d}, J=8.4 \mathrm{~Hz})$, $778(1 \mathrm{H}, \mathrm{d}, J=6.4 \mathrm{~Hz}), 7.77(1 \mathrm{H}, \mathrm{d}, J=8.4 \mathrm{~Hz})$, $7.92(1 \mathrm{H}, \mathrm{t}, J=8.0,8.8 \mathrm{~Hz}) .{ }^{13} \mathrm{C} \mathrm{NMR}\left(\mathrm{CDCl}_{3}\right) \delta$ : 
$20.44\left(\mathrm{CH}_{3}\right), 22.08\left(\mathrm{CH}_{3}\right), 29.09\left(2 \mathrm{CH}_{2}\right), 35.71(\mathrm{CH})$, $45.27\left(2 \mathrm{CH}_{2}\right), 101.03\left(\mathrm{CH}_{2}\right), 108.24(\mathrm{CH}), 109.64$ $(\mathrm{CH}), 111.21(\mathrm{CH}), 118.66(\mathrm{CH}), 121.22(\mathrm{CH}), 123.86$ $(2 \mathrm{CH}), 127.22(\mathrm{C}), 136.33(\mathrm{C}), 137.67(\mathrm{C}), 139.77(\mathrm{C})$, 140.44 (C), 141.42 (C), 148.44 (C), 149.09 (C), 172.11 (C), $173.62(\mathrm{C}=\mathrm{O})$. MS (ESI, $m / z)$ : $445.2\left[\mathrm{M}^{+}+1\right]$. Anal. calcd for $\mathrm{C}_{25} \mathrm{H}_{24} \mathrm{~N}_{4} \mathrm{O}_{4}$ (444.28): C 67.55, H 5.44, $\mathrm{N}$ 12.62; found C 67.57, H 4.41, N 12.63.

2.5r 4-(Benzo[d][1,3]dioxol-5-yl)-5-(1-acetyl-1Hindol-3-yl)-2-(1-acetylpiperidin-4-yl)oxazole (6r): Mp: 135-137 ${ }^{\circ} \mathrm{C}$. IR (KBr): 3038, 2108, 1831, 1728, 1635, $1528,1333,1244,1143,1058 .{ }^{1} \mathrm{H}$ NMR $\left(\mathrm{CDCl}_{3}\right) \delta$ : $1.45(4 \mathrm{H}, \mathrm{t}, J=2.8,1.2 \mathrm{~Hz}), 2.42(3 \mathrm{H}, \mathrm{s}), 2.73(3 \mathrm{H}, \mathrm{s})$, $2.81(1 \mathrm{H}, \mathrm{m}), 3.29(4 \mathrm{H}, \mathrm{t}, J=4.0,4.4 \mathrm{~Hz}), 6.09(2 \mathrm{H}$, s), $6.98(1 \mathrm{H}, \mathrm{d}, J=8.0 \mathrm{~Hz}), 7.10(2 \mathrm{H}, \mathrm{d}, J=6.0 \mathrm{~Hz})$, $7.22(1 \mathrm{H}, \mathrm{s}), 7.44(2 \mathrm{H}, \mathrm{t}, J=6.8,8.8 \mathrm{~Hz}), 7.61(1 \mathrm{H}$, $\mathrm{d}, J=8.0 \mathrm{~Hz}), 8.45(1 \mathrm{H}, \mathrm{d}, J=8.8 \mathrm{~Hz}) \cdot{ }^{13} \mathrm{C} \mathrm{NMR}$ $\left(\mathrm{CDCl}_{3}\right)$ \&: $21.87\left(\mathrm{CH}_{3}\right), 25.94\left(\mathrm{CH}_{3}\right), 29.33\left(2 \mathrm{CH}_{2}\right)$, $34.47(\mathrm{CH}), 44.64\left(2 \mathrm{CH}_{2}\right), 100.24(\mathrm{C}), 101.98\left(\mathrm{CH}_{2}\right)$, $108.99(\mathrm{CH}), 109.15(\mathrm{CH}), 111.47(\mathrm{CH}), 119.22(\mathrm{CH})$, $121.74(\mathrm{CH}), 122.33(\mathrm{CH}), 123.82(\mathrm{CH}), 126.35(\mathrm{C})$, $128.63(\mathrm{C}), 129.43(\mathrm{CH}), 135.23(\mathrm{C}), 138.41(\mathrm{C})$, 145.32 (C), 148.47 (C), $149.36(\mathrm{C}), 170.49(\mathrm{C}=\mathrm{O})$, $170.58(\mathrm{C}), 172.94(\mathrm{C}=\mathrm{O})$. MS (ESI, $m / z): 472.1$ $\left[\mathrm{M}^{+}+1\right]$. Anal. calcd for $\mathrm{C}_{26} \mathrm{H}_{25} \mathrm{~N}_{3} \mathrm{O}_{4}$ (471.27): $\mathrm{C}$ 68.76, H 5.36, N 8.92; found C 68.459, H 5.38, N 8.92.

2.5s 4-(Benzo[d][1,3]dioxol-5-yl)-5-(4-(piperidin-1yl)phenyl)-2-(1-acetylpiperidin-4-yl)oxazole (6s): IR $\left(\mathrm{KBr}, \mathrm{cm}^{-1}\right)$ : 3248, 3039, 2278, 2133, 1889, 1724, $1612,1574,1425,1333,1245,1144,1032,985 .{ }^{1} \mathrm{H}$ NMR $\left(\mathrm{CDCl}_{3}\right) \delta: 1.28(4 \mathrm{H}, \mathrm{m}), 1.32(4 \mathrm{H}, \mathrm{t}, J=3.6$, $2.8 \mathrm{~Hz}), 1.52(2 \mathrm{H}, \mathrm{q}), 2.34(3 \mathrm{H}, \mathrm{s}), 2.82(1 \mathrm{H}, \mathrm{m}), 3.18$ $(4 \mathrm{H}, \mathrm{t}, J=2.4,2.8 \mathrm{~Hz}), 3.57(4 \mathrm{H}, \mathrm{t}, J=2.8 .3 .2 \mathrm{~Hz})$, $6.09(2 \mathrm{H}, \mathrm{s}), 6.88(2 \mathrm{H}, \mathrm{d}, J=8.4 \mathrm{~Hz}), 6.94(1 \mathrm{H}, \mathrm{d}$, $J=7.2 \mathrm{~Hz}), 7.17(1 \mathrm{H}, \mathrm{d}, J=8.0 \mathrm{~Hz}), 7.30(1 \mathrm{H}, \mathrm{s})$, $7.74(2 \mathrm{H}, \mathrm{d}, J=8.8 \mathrm{~Hz}) .{ }^{13} \mathrm{C} \mathrm{NMR}\left(\mathrm{CDCl}_{3}\right) \delta: 21.76$ $\left(\mathrm{CH}_{3}\right), 24.54\left(2 \mathrm{CH}_{2}\right), 25.88\left(\mathrm{CH}_{2}\right), 29.36\left(2 \mathrm{CH}_{2}\right)$, $35.87(\mathrm{CH}), 45.66\left(2 \mathrm{CH}_{2}\right), 55.28\left(\mathrm{CH}_{2}\right), 101.09\left(\mathrm{CH}_{2}\right)$, $108.55(\mathrm{CH}), 109.66(\mathrm{CH}), 113.33(2 \mathrm{CH}), 119.78(\mathrm{C})$, $121.36(\mathrm{CH}), 127.44(2 \mathrm{CH}), 128.09(\mathrm{C}), 137.22(\mathrm{C})$, 146.08 (C), 148.22 (C), 149.19 (C), 150.77 (C), 171.22 (C), $173.66(\mathrm{C}=\mathrm{O})$. MS (ESI, $m / z)$ : $474.2\left[\mathrm{M}^{+}+1\right]$. Anal. calcd for $\mathrm{C}_{28} \mathrm{H}_{31} \mathrm{~N}_{3} \mathrm{O}_{4}$ (473.56): C 71.04, H 6.69, $\mathrm{N}$ 8.81; found C 71.07, H 6.72, N 8.86.

2.5t 4-(Benzo[d][1,3]dioxol-5-yl)-5-(4-morpholinophenyl)-2-(1-acetylpiperidin-4-yl)oxazole (6t): $\mathrm{IR}(\mathrm{KBr}$, $\left.\mathrm{cm}^{-1}\right)$ : 3305, 3007, 2225, 2143, 1851, 1733, 1645, 1534, 1338, 1284, 1112, 963, 854. ${ }^{1} \mathrm{H}$ NMR $\left(\mathrm{CDCl}_{3}\right)$ $\delta: 1.42(4 \mathrm{H}, \mathrm{t}, J=2.8,2.0 \mathrm{~Hz}), 2.37(3 \mathrm{H}, \mathrm{s}), 2.81(1 \mathrm{H}$, $\mathrm{m}), 3.14(4 \mathrm{H}, \mathrm{t}, J=4.8,1.6 \mathrm{~Hz}), 3.35(4 \mathrm{H}, \mathrm{t}, J=$ 2.0, $2.4 \mathrm{~Hz}), 3.68(4 \mathrm{H}, \mathrm{t}, J=4.4,4.8 \mathrm{~Hz}), 6.11(2 \mathrm{H}$, s), $6.86(2 \mathrm{H}, \mathrm{d}, J=6.4 \mathrm{~Hz}), 6.98(1 \mathrm{H}, \mathrm{d}, J=8.8 \mathrm{~Hz})$, $7.19(1 \mathrm{H}, \mathrm{d}, J=8.8 \mathrm{~Hz}), 7.32(1 \mathrm{H}, \mathrm{s}), 7.65(2 \mathrm{H}, \mathrm{d}, J$ $=8.0 \mathrm{~Hz}) \cdot{ }^{13} \mathrm{C} \mathrm{NMR}\left(\mathrm{CDCl}_{3}\right) \delta: 21.88\left(\mathrm{CH}_{3}\right), 29.74$ $\left(2 \mathrm{CH}_{2}\right), 35.16(\mathrm{CH}), 45.12\left(2 \mathrm{CH}_{2}\right), 55.99\left(\mathrm{CH}_{2}\right), 66.38$ $\left(2 \mathrm{CH}_{2}\right), 101.12\left(\mathrm{CH}_{2}\right), 108.24(\mathrm{CH}), 109.62(\mathrm{CH})$, $113.12(2 \mathrm{CH}), 119.52(\mathrm{C}), 121.74(\mathrm{CH}), 127.63(2 \mathrm{CH})$, 128.12 (C), 137.27 (C), 146.18 (C), 148.78 (C), 149.34 (C), $150.54(\mathrm{C}), 171.24(\mathrm{C}), 173.67(\mathrm{C}=\mathrm{O})$. MS (ESI, $m / z)$ : $476.1\left[\mathrm{M}^{+}+1\right]$. Anal. calcd for $\mathrm{C}_{27} \mathrm{H}_{29} \mathrm{~N}_{3} \mathrm{O}_{5}$ (475.54): C 68.17, H 6.17, N 8.80; found C 68.17, H $6.12, \mathrm{~N} 8.83$.

\subsection{Synthesis of compound (7)}

A well-stirred solution of compound 1 (1 eq.) in $\mathrm{AcOH}$ was treated with 1,3-propanedithiol (2 eq.) and $\mathrm{BF}_{3} \mathrm{OEt}_{2}$ (2.5 eq.) at $0^{\circ} \mathrm{C}$. The mixture was warmed to room temperature and stirred for $2 \mathrm{~h}$. The solid that precipitated was filtered, washed with cold $\mathrm{H}_{2} \mathrm{O}$ and dried under reduced pressure to give compound 7 in $87 \%$ yield. Mp: $98-101^{\circ} \mathrm{C}$. IR $\left(\mathrm{KBr}, \mathrm{cm}^{-1}\right): 3012,2142$, $1618,1506,1341,1207,1122 .{ }^{1} \mathrm{H}$ NMR $\left(\mathrm{CDCl}_{3}\right) \delta$ : $1.82\left(2 \mathrm{H}, \mathrm{q}, \mathrm{CH}_{2}\right), 2.85(2 \mathrm{H}, \mathrm{t}, J=4.8,4.0 \mathrm{~Hz}), 3.03$ $(2 \mathrm{H}, \mathrm{t}, J=4.8 \mathrm{~Hz}), 5.13(1 \mathrm{H}, \mathrm{s}), 5.98(2 \mathrm{H}, \mathrm{s}), 6.76(1 \mathrm{H}$, $\mathrm{d}, J=8.8 \mathrm{~Hz}), 6.98(1 \mathrm{H}, \mathrm{d}, J=8.0 \mathrm{~Hz}), 7.24(1 \mathrm{H}$, s). ${ }^{13} \mathrm{C} \mathrm{NMR}\left(\mathrm{CDCl}_{3}\right) \delta: 25.05\left(\mathrm{CH}_{2}\right), 27.55\left(2 \mathrm{CH}_{2}\right)$, $51.75(\mathrm{CH}), 102.45\left(\mathrm{CH}_{2}\right), 103.77(\mathrm{CH}), 104.41(\mathrm{CH})$, $122.34(\mathrm{CH}), 132.15$ (C), 147.92 (C), 148.01 (C). MS (ESI, $m / z$ ): $241.2\left[\mathrm{M}^{+}+1\right]$. Anal. calcd for $\mathrm{C}_{11} \mathrm{H}_{12} \mathrm{O}_{2} \mathrm{~S}_{2}$ (240.05): C 54.96, H 5.03, S 26.65; found C 54.97, H $5.04, \mathrm{~S} 26.65$.

\subsection{General procedure for the synthesis of compound ( 8 )}

Freshly titrated $n$-BuLi (1 M in hexane, 1.2 eq.) was added drop-wise to a solution of 7 (1 eq.) in THF at $-78^{\circ} \mathrm{C}$ under inert atmosphere. After stirring at $-78^{\circ} \mathrm{C}$ temperature for $30 \mathrm{~min}$ respective aldehydes (1 eq.) was added to the above reaction mixture at $-78^{\circ} \mathrm{C}$. The resulting mass was warmed to room temperature and stirred for further $2-3 \mathrm{~h}$. The reaction was quenched with saturated aqueous $\mathrm{NH}_{4} \mathrm{Cl}$ solution and extracted with EtOAc. The organic extract was washed with brine, dried over anhydrous $\mathrm{Na}_{2} \mathrm{SO}_{4}$ and concentrated under reduced pressure. The residue obtained was purified by CC [silica gel (60-120) EtOAc-petroleum] to get pure compound $\mathbf{8}$ in $79-88 \%$ yield. 
2.7a 2-(Benzo[d][1,3]dioxol-5-yl)-1,3-dithian-2-yl) furon-2-yl)methanol $(\boldsymbol{8 b})$ : Yield: 85\%. $\mathrm{mp}$ : 173$175^{\circ} \mathrm{C}$. IR $\left(\mathrm{KBr}, \mathrm{cm}^{-1}\right): 3321,3124,2105,1709,1654$, $1536,1375,1200,1112,1005 .{ }^{1} \mathrm{H}$ NMR $\left(\mathrm{CDCl}_{3}\right) \delta$ : $1.24(2 \mathrm{H}, \mathrm{q}), 1.52(1 \mathrm{H}, \mathrm{s}), 1.98(2 \mathrm{H}, \mathrm{t}, J=4.4,4.8 \mathrm{~Hz})$, $2.62(2 \mathrm{H}, \mathrm{t}, J=2.4,2.0 \mathrm{~Hz}), 5.01(1 \mathrm{H}, \mathrm{s}), 5.94(2 \mathrm{H}, \mathrm{s})$, $6.15(1 \mathrm{H}, \mathrm{d}, J=8.8 \mathrm{~Hz}), 6.24(1 \mathrm{H}, \mathrm{d}, J=6.4 \mathrm{~Hz}), 6.74$ $(1 \mathrm{H}, \mathrm{d}, J=8.0 \mathrm{~Hz}), 6.99(1 \mathrm{H}, \mathrm{d}, J=8.4 \mathrm{~Hz}), 7.24(1 \mathrm{H}$, s), $7.32(1 \mathrm{H}, \mathrm{t}, J=4.0,4.4 \mathrm{~Hz}) \cdot{ }^{13} \mathrm{C} \mathrm{NMR}\left(\mathrm{CDCl}_{3}\right)$ $\delta$ : $25.35\left(\mathrm{CH}_{2}\right), 26.58\left(2 \mathrm{CH}_{2}\right), 69.75(\mathrm{C}), 90.55(\mathrm{CH})$, $102.55\left(\mathrm{CH}_{2}\right), 109.82(\mathrm{CH}), 111.70(\mathrm{CH}), 112.44(\mathrm{CH})$, $113.05(\mathrm{CH}), 123.08(\mathrm{CH}), 132.45(\mathrm{C}), 141.86(\mathrm{CH})$, 147.84 (C), 148.26 (C), 153.75 (C). MS (ESI, $m / z$ ): $337.2\left[\mathrm{M}^{+}+1\right]$. Anal. calcd for $\mathrm{C}_{16} \mathrm{H}_{16} \mathrm{O}_{4} \mathrm{~S}_{2}$ (336.13): $\mathrm{C}$ 57.16, H 4.78, S 19.05; found C 57.17, H 4.74, S 19.05.

\subsection{General procedure for the synthesis of compound (4)}

A suspension of compound $\mathbf{8}$ (1 eq.) in $90 \%$ aqueous $\mathrm{MeOH}$ was treated with $\mathrm{HgCl}_{2}$ (2.1 eq.) and $\mathrm{HgO}$ (1.3 eq, red form) at room temperature. The reaction mixture was refluxed for $3-4 \mathrm{~h}$. The reaction mixture was cooled to room temperature, filtered the salts and washed with $\mathrm{CH}_{2} \mathrm{Cl}_{2}$. The combined filtrate was concentrated under reduced pressure to give a residue. The residue was diluted with $\mathrm{CH}_{2} \mathrm{Cl}_{2}$, washed with water and brine solution. After drying over anhydrous $\mathrm{Na}_{2} \mathrm{SO}_{4}$ and concentrated under reduced pressure to give crude compound 4 in $78-82 \%$ yields.

\section{Results and discussion}

The sequence leading to the formation of the title compounds is outlined in scheme 1. A solution of benzo[ $d][1,3]$ dioxole-5-carbaldehyde (1) in $\mathrm{MeCN}$ was added drop-wise to a stirring mixture of $\mathrm{KCN}, \mathrm{TBSCl}$ and $\mathrm{ZnI}_{2}$ at room temperature for overnight to afford compound $2{ }^{20-22}$ as pale brown solid in $85 \%$ yield, which on treatment with aldehydes in the presence of litiumdiisopropylamide (LDA) to yielded $\mathbf{3}^{23}$ with good yields. The structure of these products was deduced from their elemental analysis, IR ${ }^{1} \mathrm{H}$ - and ${ }^{13} \mathrm{C}$-NMR and MS data. These compounds were allowed to react with $10 \% \mathrm{HCl}$ in $\mathrm{THF}$ to afford the intermediates 4. The subsequent coupling of $\mathbf{4}$ with 1-acetylpiperidine4-carboxylic acid yielded $\mathbf{5}$, which on treatment with $\mathrm{NH}_{4} \mathrm{OAc}$, in $\mathrm{AcOH}$ gave multi-substituted oxazole derivatives 6a-6t. The intermediate $\mathbf{4}$ was also obtained via the synthesis of compound $\mathbf{7}$ and $\mathbf{8}$ (scheme 1). ${ }^{24}$ The structures of the isolated products $\mathbf{6 a}-\mathbf{6 t}$ were

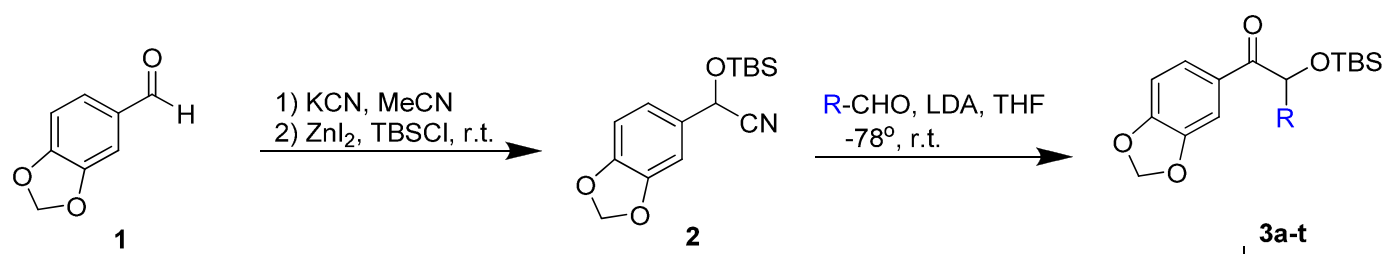

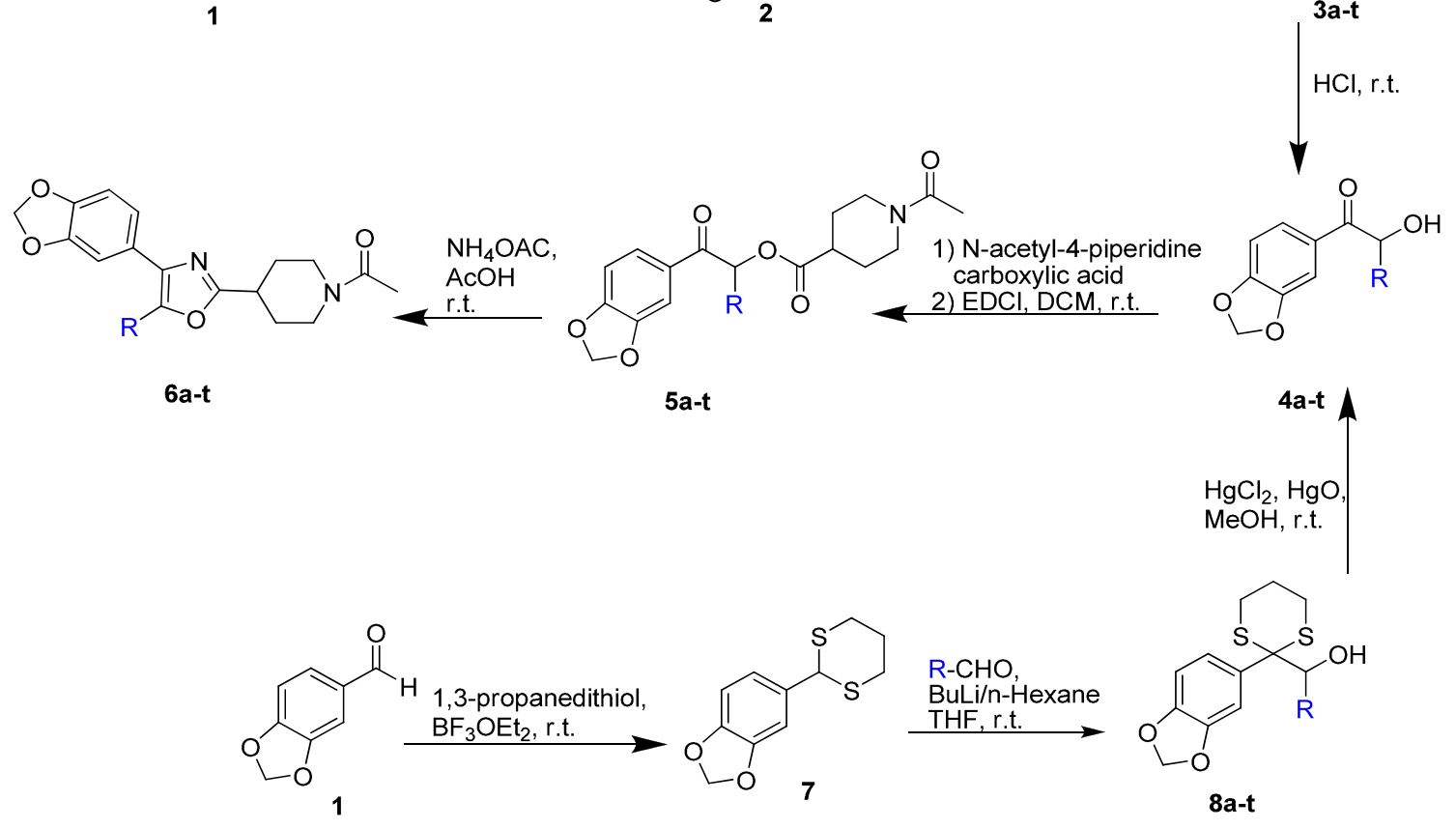

Scheme 1. Synthesis of a new class of multi-substituted oxazole derivatives. 


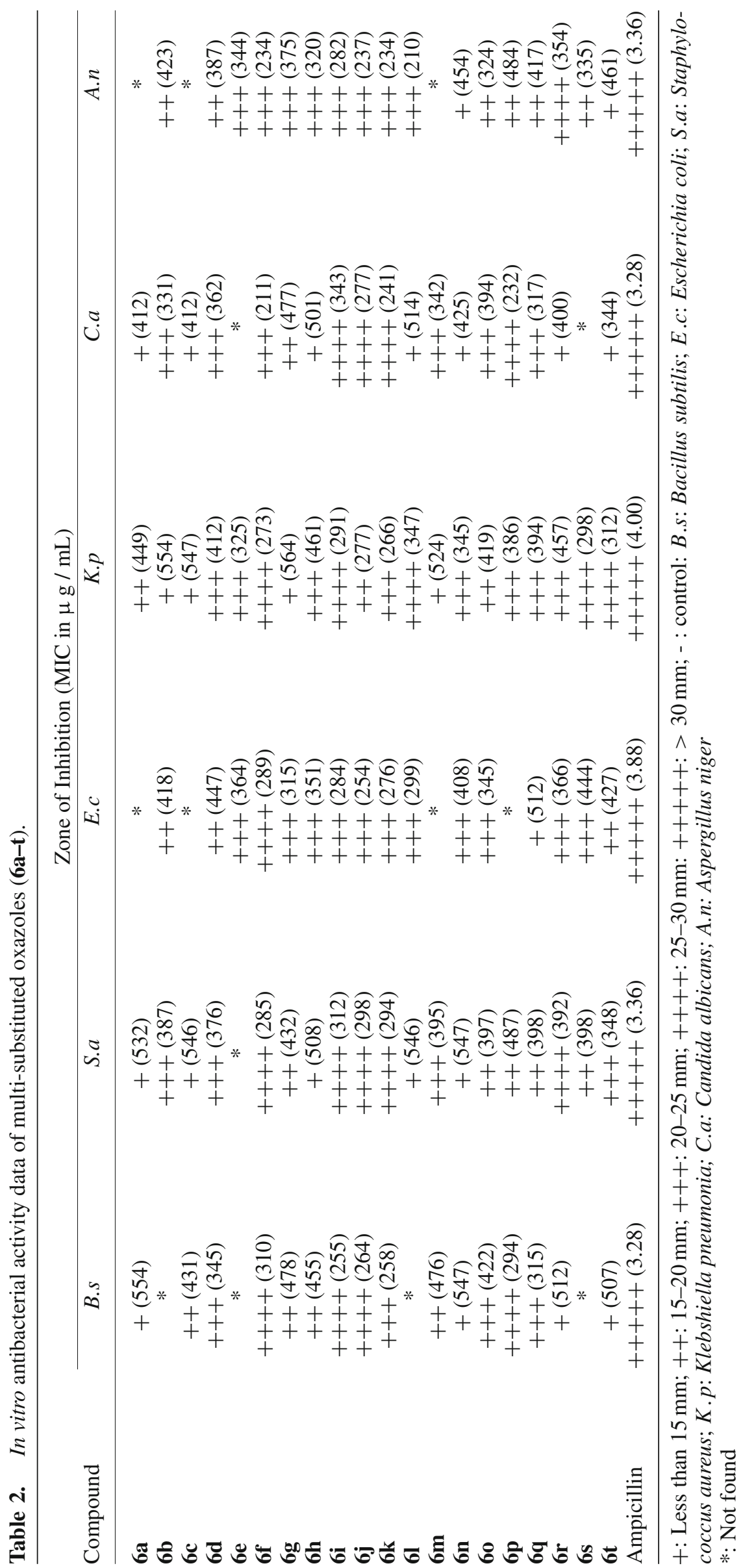


deduced from their elemental analyses, IR, ${ }^{1} \mathrm{H},{ }^{13} \mathrm{C}$ NMR and MS spectra.

\section{Biological activity}

It is reported ${ }^{25,26}$ that oxazoles showed antimicrobial activity against (Staphylococcus aureus; Escherichia coli; Bacillus subtilis; Klebshiella pneumonia). In view of that, in order to search for new antimicrobial agents, all the twenty compounds were evaluated for their antimicrobial activity against the above four bacteria and two fungi. The investigation of antimicrobial activity (tables 2 ) revealed that all the newly synthesized compounds showed good to moderate minimum inhibitory concentration at $258-564 \mu \mathrm{g} / \mathrm{mL}$ in $20 \%$ water in dimethyl sulphoxide. Out of the twenty compounds, $\mathbf{6 f}, \mathbf{6 j}, \mathbf{6} \mathbf{i}$ and $\mathbf{6 k}$ exhibited pronounced antibacterial activity, and the inhibitory activity among these four compounds followed the increasing order: $\mathbf{6 k}>\mathbf{6 j}>\mathbf{6 i}>\mathbf{6 f}$. Compounds $6 \mathbf{g}, \mathbf{6 h}, \mathbf{6 0}$ and $\mathbf{6 r}$ showed good activity against Escherichia coli. Compounds 6s and 6t showed good activity against Klebshiella pneumonia. Compounds $\mathbf{6 p}$ and $\mathbf{6 q}$ moderate activity against Bacillus subtilis. The compounds were also screened for their antifungal activity against Candida albicans and Aspergillus niger using the fungicide clotrimazole in DMF as a standard. ${ }^{27}$

\section{Conclusion}

In this work, we have reported a new class of multisubstituted oxazole derivatives which are characterized by FT-IR, PMR and ${ }^{13} \mathrm{C}$ NMR spectral analysis. Synthesized compounds are screened for their antifungal and antibacterial activity. These compounds show good activity against Gram + ve and Gram -ve bacteria and fungal species.

\section{Acknowledgement}

The authors are thankful to Sri Krishnadevaraya University for supporting this work.

\section{References}

1. Palmer D C (ed.) 2003 Oxazoles: Synthesis, reactions and spectroscopy, Part A and Part B; Hoboken NJ: John Wiley \& Sons, 331

2. (a) Jin Z 2006 Nat. Prod. Rep. 23 464; (b) Yeh V S C 2004 Tetrahedron 6011995

3. Wipf P 1995 Chem. Rev. 952115

4. Wipf P and Yokokawa F 1998 Tetrahedron Lett. 392223

5. Nishida A, Fuwa M, Naruto S, Sugano Y, Saito H and Nakagawa M 2000 Tetrahedron Lett. 414791

6. Williams D R and Fu L 2010 Synlett 40591

7. Lai P S and Taylor M S 2010 Synthesis 91449

8. Wu B, Wen J, Zhang J, Li J, Xiang Y-Z and Yu X-Q 2009 Synlett 30500

9. Lee K, Counceller C M and Stambuli J P 2009 Org. Lett. 61457

10. Misra N C and Ila H 2010 J. Org. Chem. 755195

11. Keni M and Tepe J J 2005 J. Org. Chem. 704211

12. Takeuchi H, Yanagida $S$, Ozaki T, Hagiwara $S$ and Eguchi S 1989 J. Org. Chem. 54431

13. Pei W, Li S, Nie X, Li Y, Pei J, Chen B, Wu J and Ye X 1998 Synthesis 1298

14. Ang K H, Prager R H, Smith J A, Weber B and Williams C M 1996 Tetrahedron Lett. 37, 675

15. Prager R H, Smith J A, Weber B and Williams C M 1997 J. Chem. Soc., Perkin Trans. 12659

16. Lee J C and Hong T 1997 Tetrahedron Lett. 388959

17. Varma R S and Kumar D 1998 J. Heterocycl. Chem. 35 1533

18. Graham T H 2010 Org. Lett. 163614

19. Barrett A G M, Cramp S M, Hennessy A J, Procopiou P A and Roberts R S 2002 Org. Lett. 2271

20. Pamela M, Shijie Z and Lanny L 1999 J. Org. Chem. 64 2145

21. Rawa V H, Appa Rao J and Cava M P 1985 Tetrahedron Lett. 4275

22. Xin L, Cory B and Jeffrey S J 2005 J. Am. Chem. Soc. 1271833

23. Toray Industries, Inc. 2008 Patent: EP2009006 A1, 110

24. Rozwadowska M D and Chrzanowska M 1985 Tetrahedron 142885

25. (a) Mohamed K, Venugopala K, Narayanaswamy B, Mohana Raju A and Gopal Krishna Rao 2009 Lett. Drug Des. Discov. 6 21-28; (b) Tejani S and Sanoski C 2009 Davis's pocket clinical drug reference, F A Davis Company, 266

26. Oludotun A P, Edet E U and Santhosh M S 2008 Eur. J. Med. Chem. 431095

27. British Pharmacopoeia 1953 London: Pharmaceutical Press, pp 796 